Supporting Information for

\title{
Regioselective Synthesis of Substituted Pyrroles: Efficient Palladium-Catalyzed Cyclization of Internal Alkynes and 2-Amino-3-Iodoacrylate Derivatives
}

\author{
Matthew L. Crawley, ${ }^{1}$ Igor Goljer, ${ }^{2}$ Douglas J. Jenkins, ${ }^{1}$ John F. Mehlmann, ${ }^{1}$ Lisa Nogle, ${ }^{2}$ \\ Rebecca Dooley, ${ }^{2}$ and Paige Mahaney ${ }^{1}$ \\ ${ }^{1}$ Discovery Medicinal Chemistry and ${ }^{2}$ Discovery Analytical Chemistry, Wyeth Research, 500 \\ Arcola Road, Collegeville, PA 19426.
}

\section{SPECTRA}


<smiles>COC(=O)/C(=C/I)NC(C)=O</smiles>

Tube \#1963 in position $12 \mathrm{Jul} \mathrm{26,2006}$ Bopt I H1 32 ocane in dmeo

pad-10 run with gradahim before acquiaition

Aquiaition finiahed at 10.37 BDT on Wod Jul 26, 2006

Automation directory1/export/home/vami/vnmroys/autorun/

File 1202

Pulvo Sequenoel a2pul

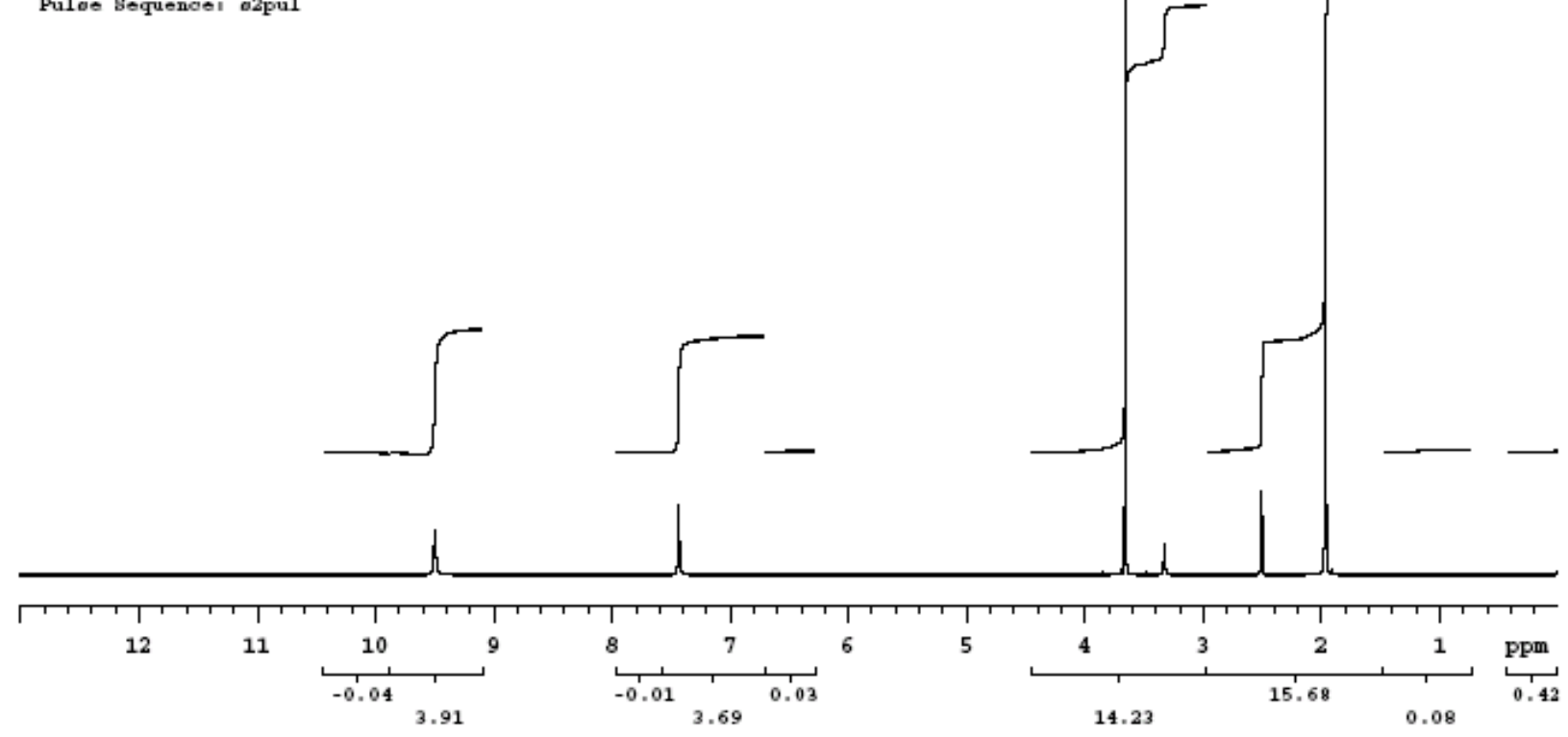

Tube \# 1963 in position $12 \mathrm{Jul} \mathrm{26,2006}$

Bupt, Quisk $\mathrm{Cl}_{3}$ in dmeo

Aquiaition finiohed at 10,41 BDT on Wod Jul 26, 2006

Automation directory1/export/hcme/vnmr1/vnmreys/autorun/

File , 1203

Puloe Sequencel a2pul

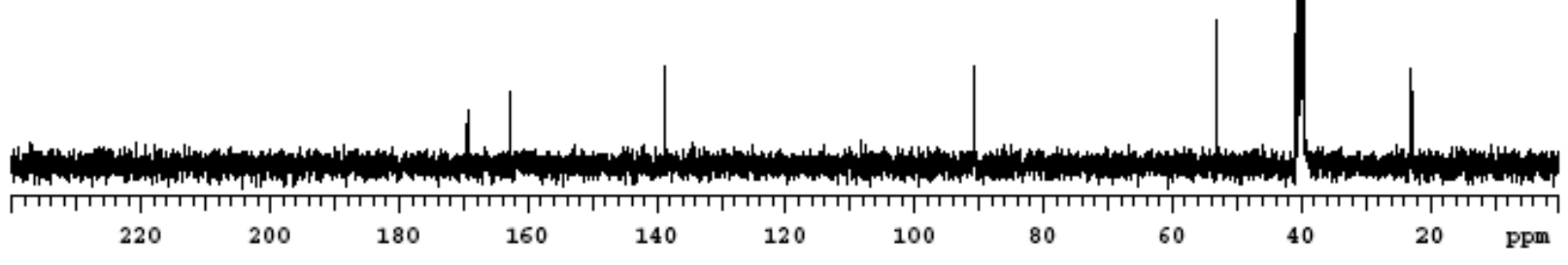




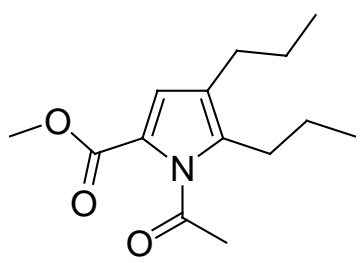

$4 a$

Tube \# 1994 in poaition 1 Jul 26, 2006 Expt: H1 32 coane in twoo

pad-10 run with gradohin berore acquideition

Aquiation finiabed at 17:14 EDT on พed Jul 26, 2006

Autonation directory: /export/hone/vnnr1/vnmroye/autorun/

File : 0104

Dulae sequence: arpul

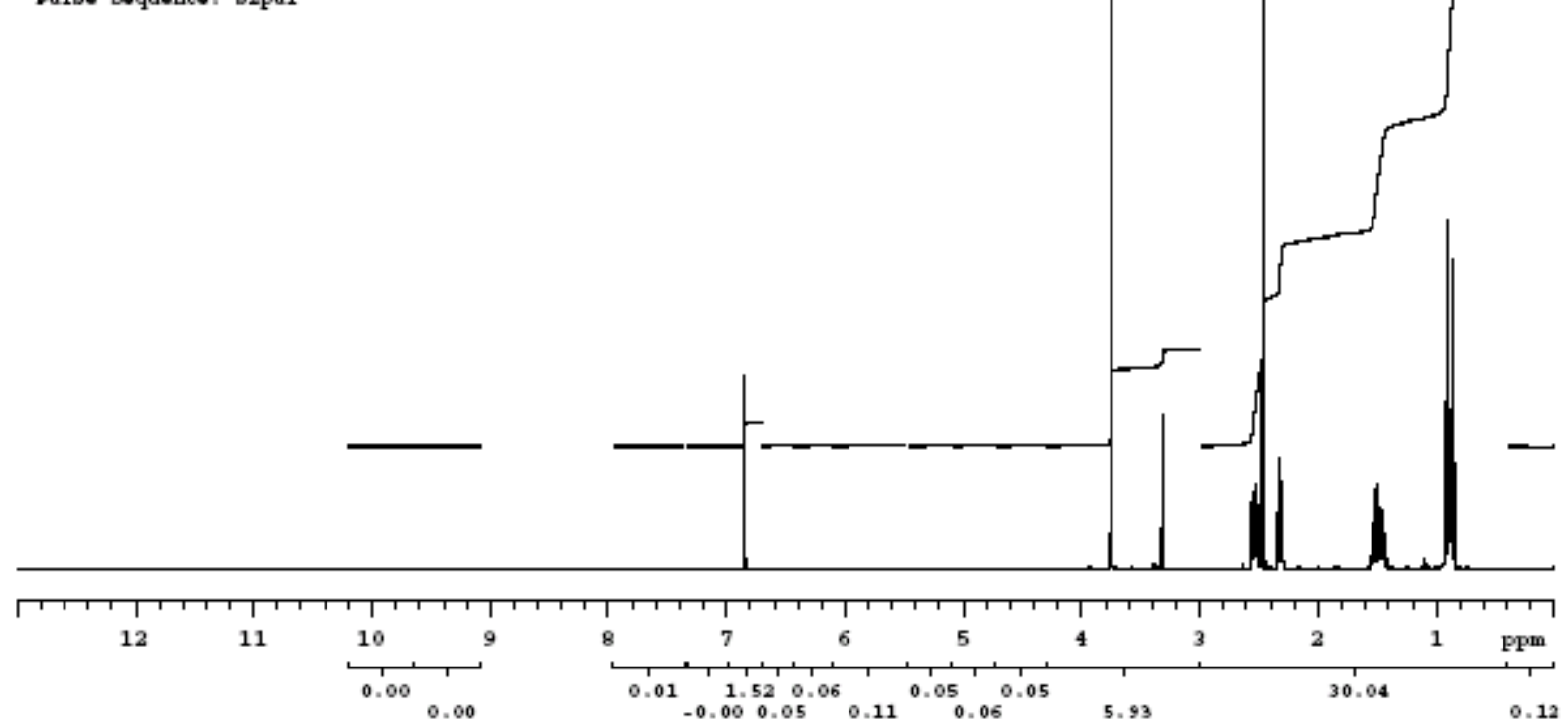

Tube \# 1270 in position $14 \mathrm{Jul} \mathrm{05,2006}$

Bupt I Quisk $\mathrm{C} 13$ in dmeo

pad-10 run with gradahim bofore acquiaition

Aquiaition finiohed at 11,26 BDT on Wod Jul 05, 2006

Automation direotory'/export/hcmo/vnmr1/vnmreys/autorun/ File, 1402

Puloo Soquenool a2pul

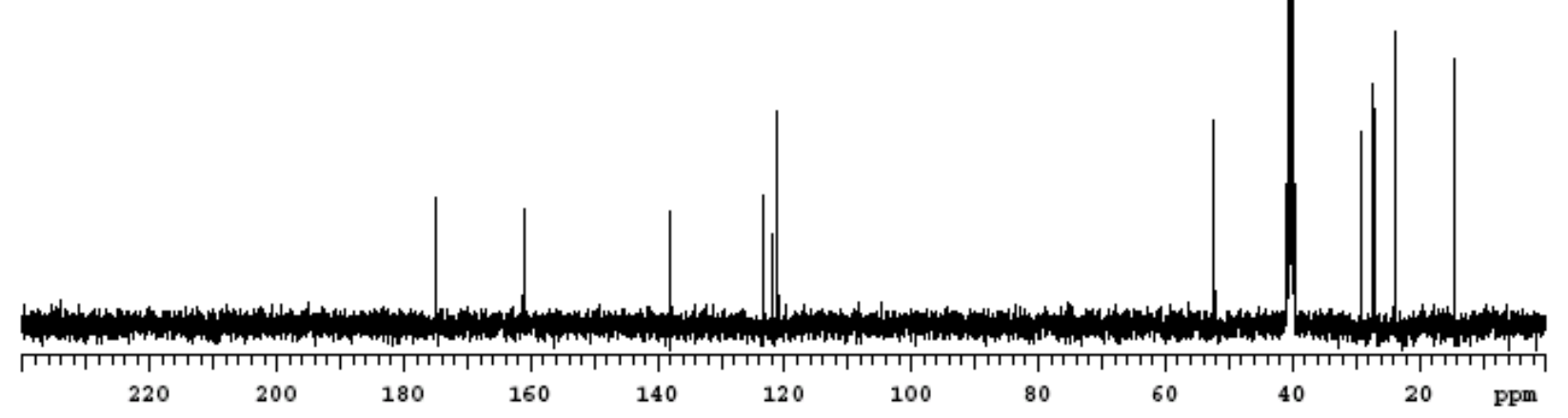




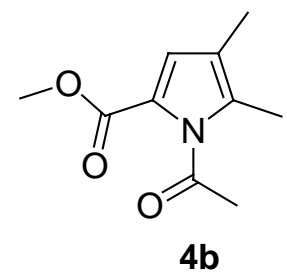

Tube \#2166 in position 14 Aug 01, 2006

Bopt I H1 32 ecane in dmeo

pad-10 run with gradahim bofore acquioition

Aquioition finiohed at 13,33 BDT on Tus Aug 01, 2006

Automation directoryl/export/homo/vnmr1/vnmroys/autorun/

File I 1406

Puleo Sequenoel a2pul

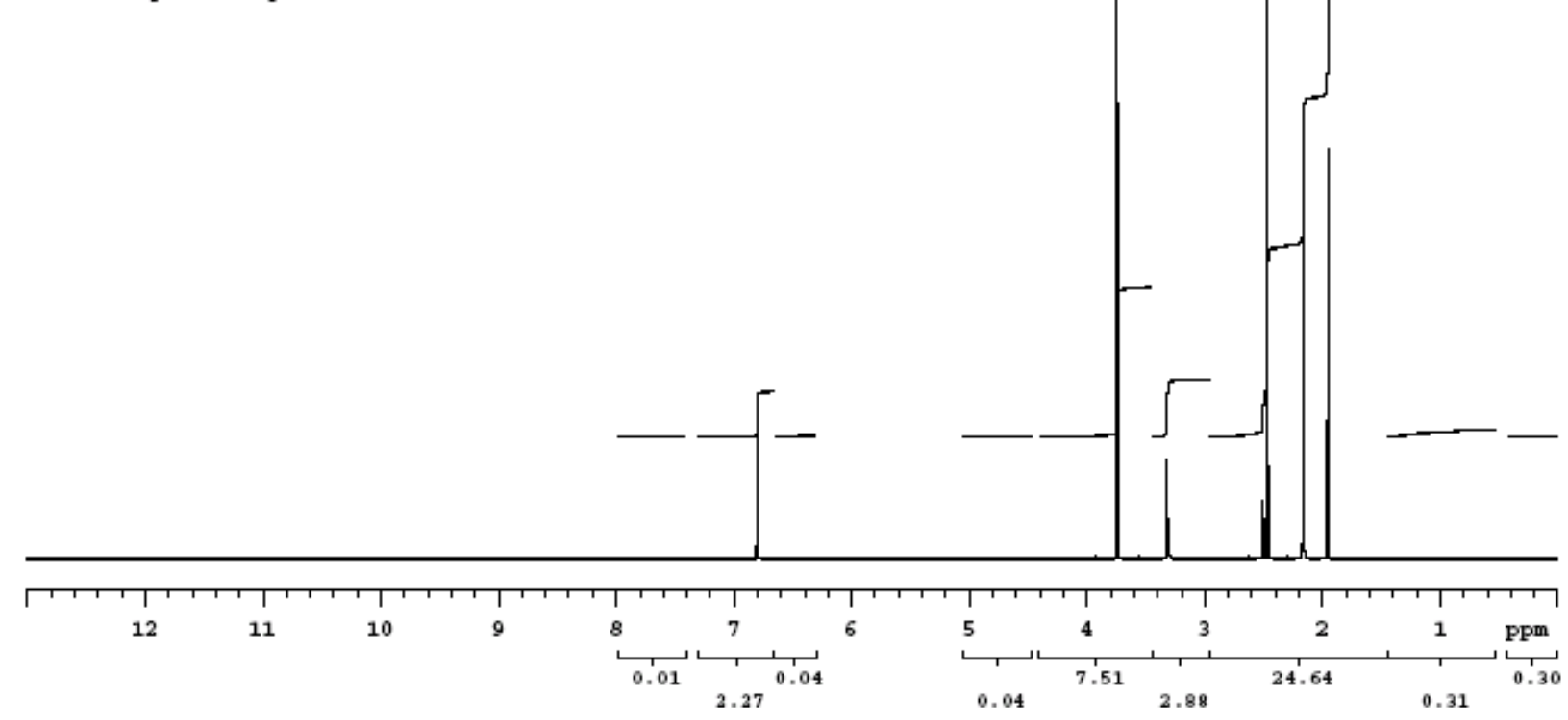

Tube $\$ 2217$ in position 14 Aug 02,2006

Bopt, Quisk $\mathrm{C} 13$ in dmeo

Aquiaition finiohed at 14,27 EDT on Wod Aug 02, 2006

Automation directory'/export/hcme/vnmi1/vnmege/autorun/

File I 1404

Pulao Soquenoel a2pul

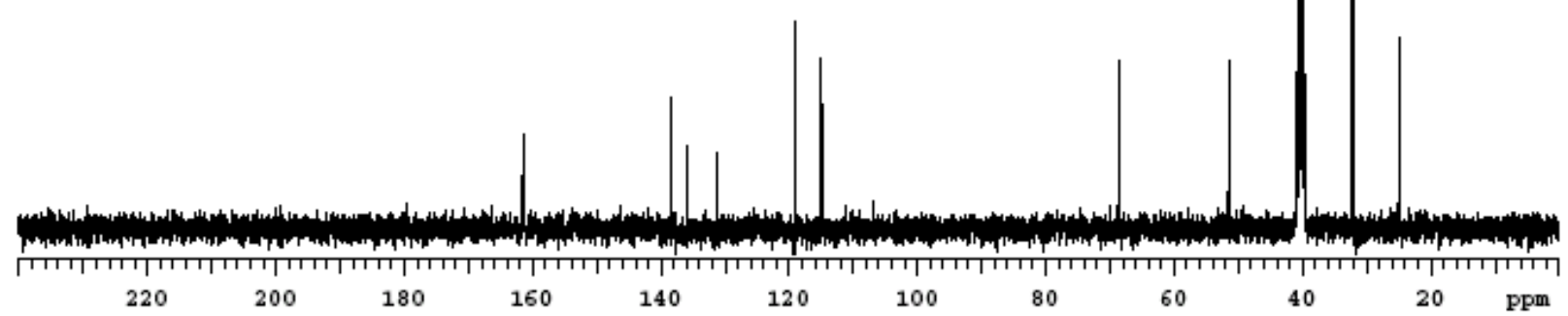




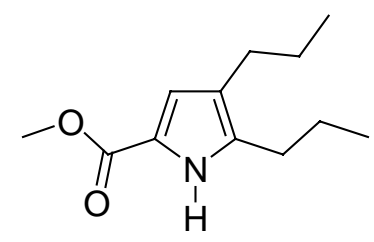

Tube \# 2312 in position 12 Aug 04, 2006

Bopt, H1 32 ecane in dmeo

pad-10 run with gradshim before acquioition

Aquioition finiohed at 14,33 BDT on Pri Aug 04, 2006

Automation directoryı/export/hcme/vnmx1/vnmegys/autorun/

File, 1204

Puloo Soquenool a2pul

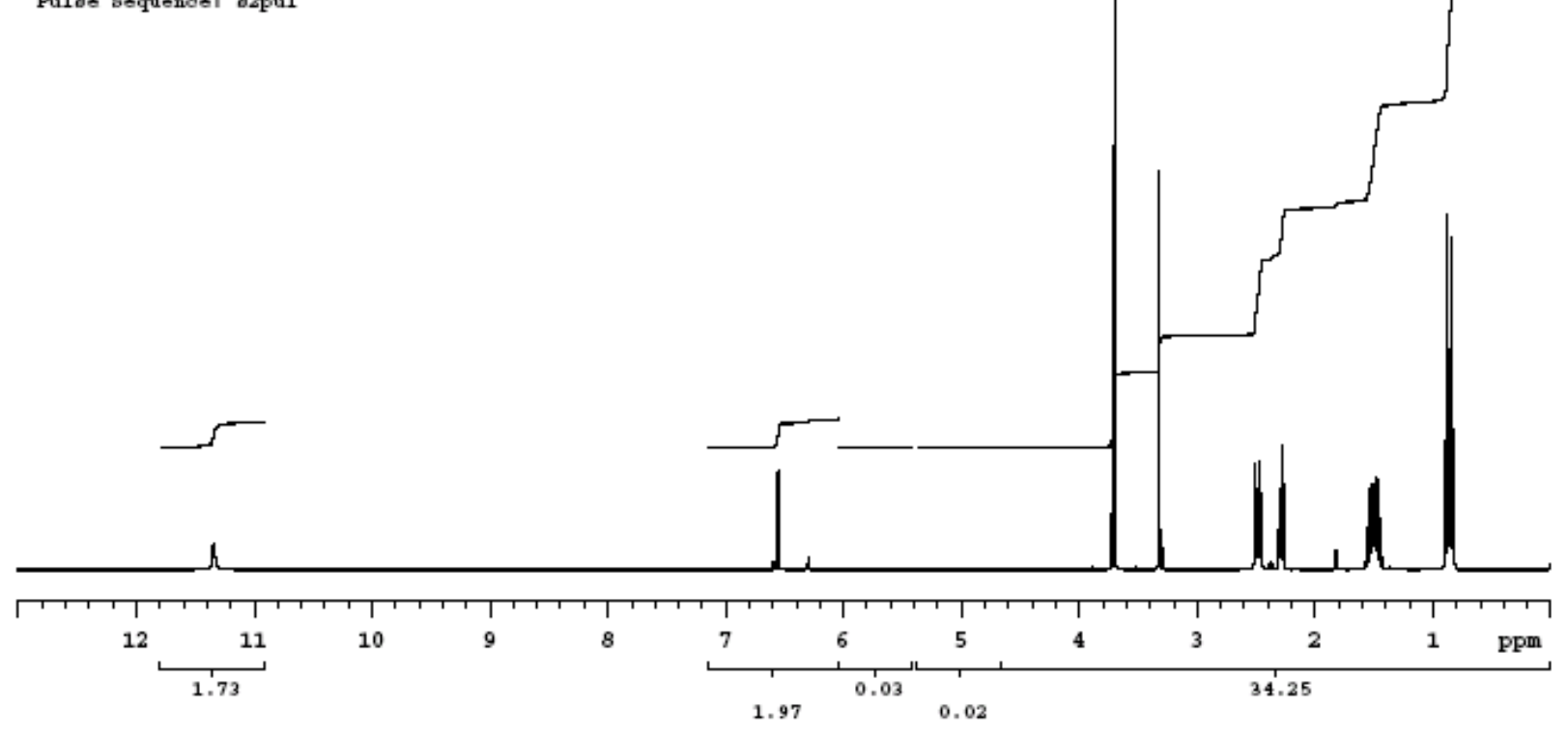

Tube \#2312 in position 12 Aug 04, 2006

Bropt I Quiok C13 in dmeo

Aquiaition finiohed at 14,37 EDT on Pri Aug 04, 2006

Automation directoryı/export/hcme/vnmr1/vnmreys/autorun/

File , 1204

Pulae Sequencer a2pul

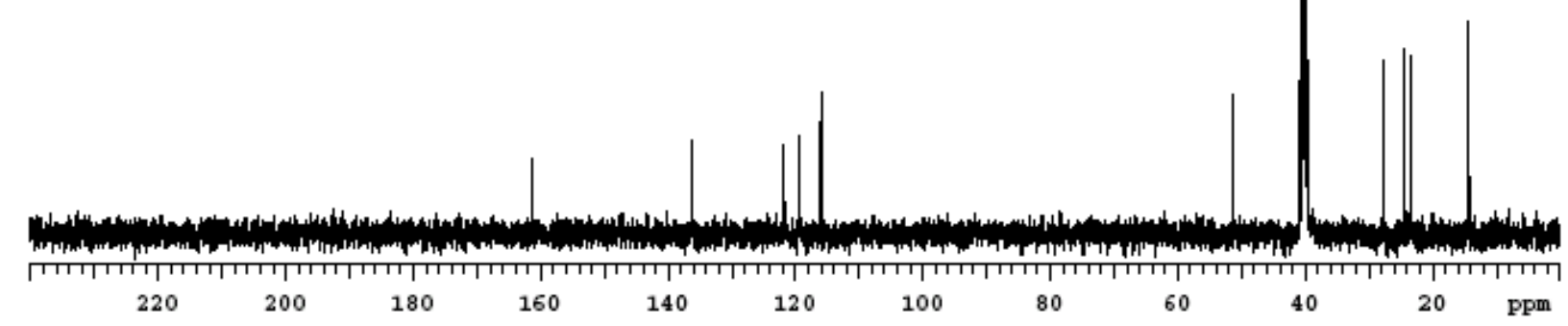




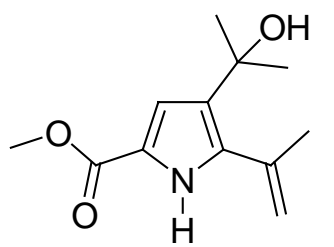

$5 d$

Tube \# 2217 in position 14 Aug 02,2006

Bupt, H1 32 ecane in dmeo

pad-10 run with gradahim before acquiaition

Aquiaition finiohed at 14,22 BDT on Wod Aug 02, 2006

Automation directoryl/export/home/vnmi/vnmroys/autorun/

File, 1403

Pulve Sequenoel a2pul

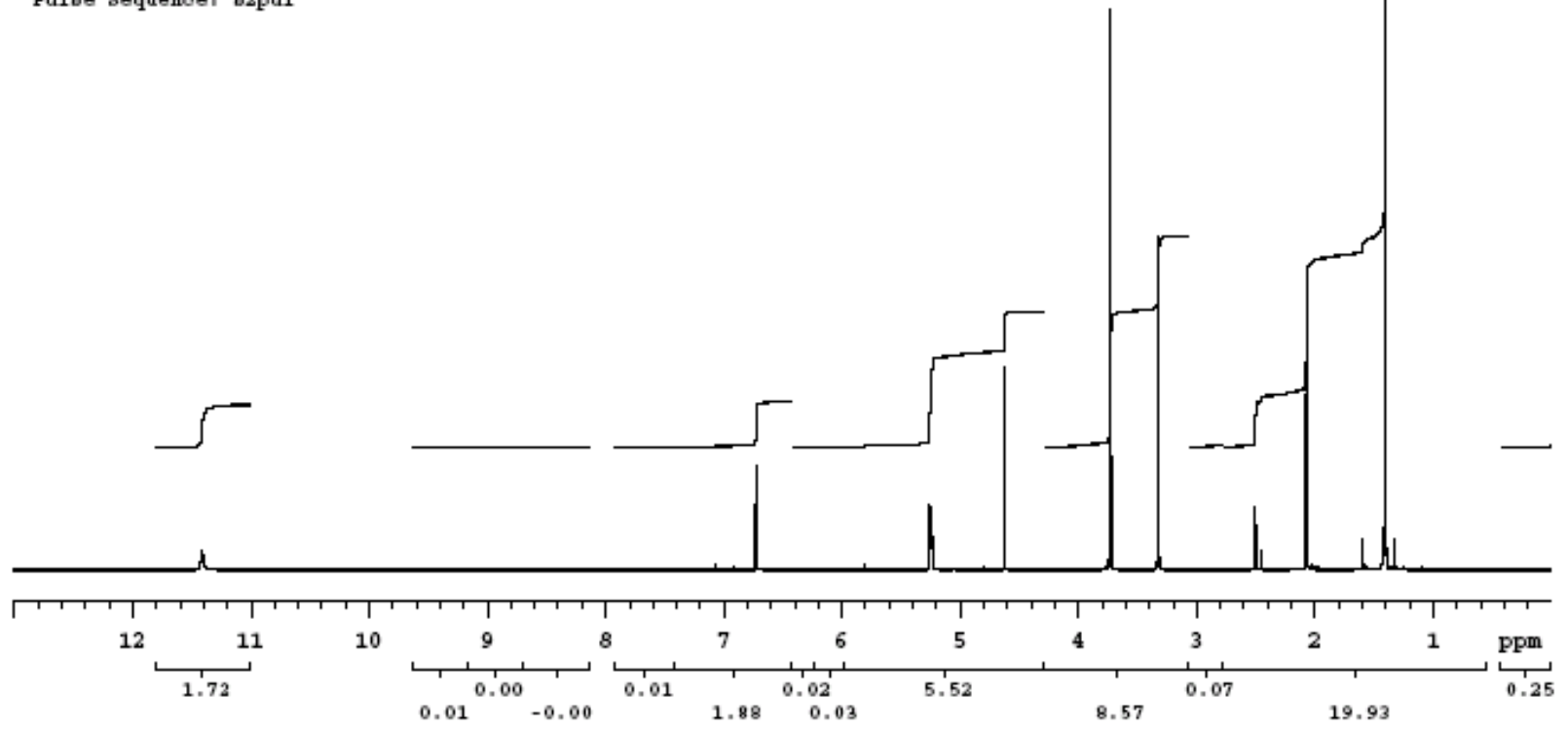

Tube \#2217 in position 14 Ang 02, 2006

Brpt, Quiok $\mathrm{C} 13$ in dmeo

Aquiaition finiohed at 14,27 EDT on Wed Aug 02, 2006

Automation directoryı/export/hcmo/vnmr1/vnmroys/autorun/

File I 1404

Puleo Sequencel a2pul

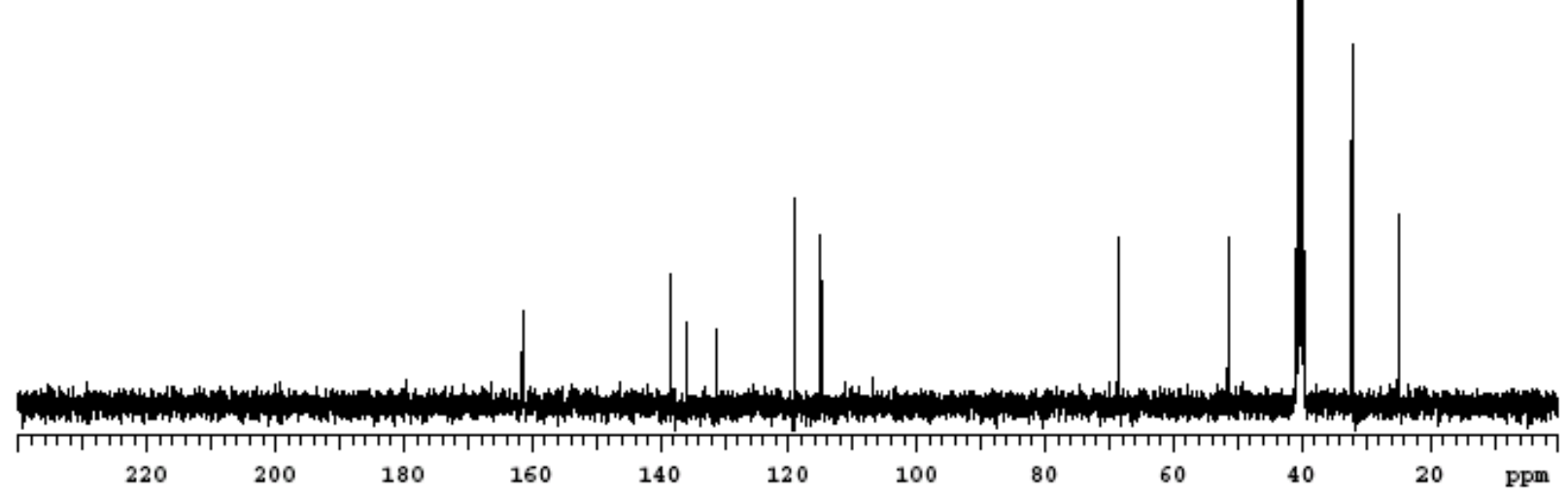




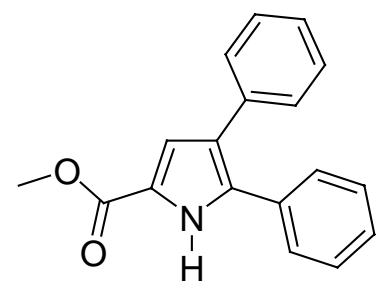

$5 e$

Tube \#2134 in position 7 Jul 31, 2006 Bropt, H1 32 ecane in dmeo

pad-10 run with gradahim before acquiaition Aquiaition finiohed at 17,43 BDT on Hon Jul 31, 2000

Automation directory/ /export/homo/vamr1/vnmroys/a utorun/ File I 0704

Puloo Sequenoer o2pul
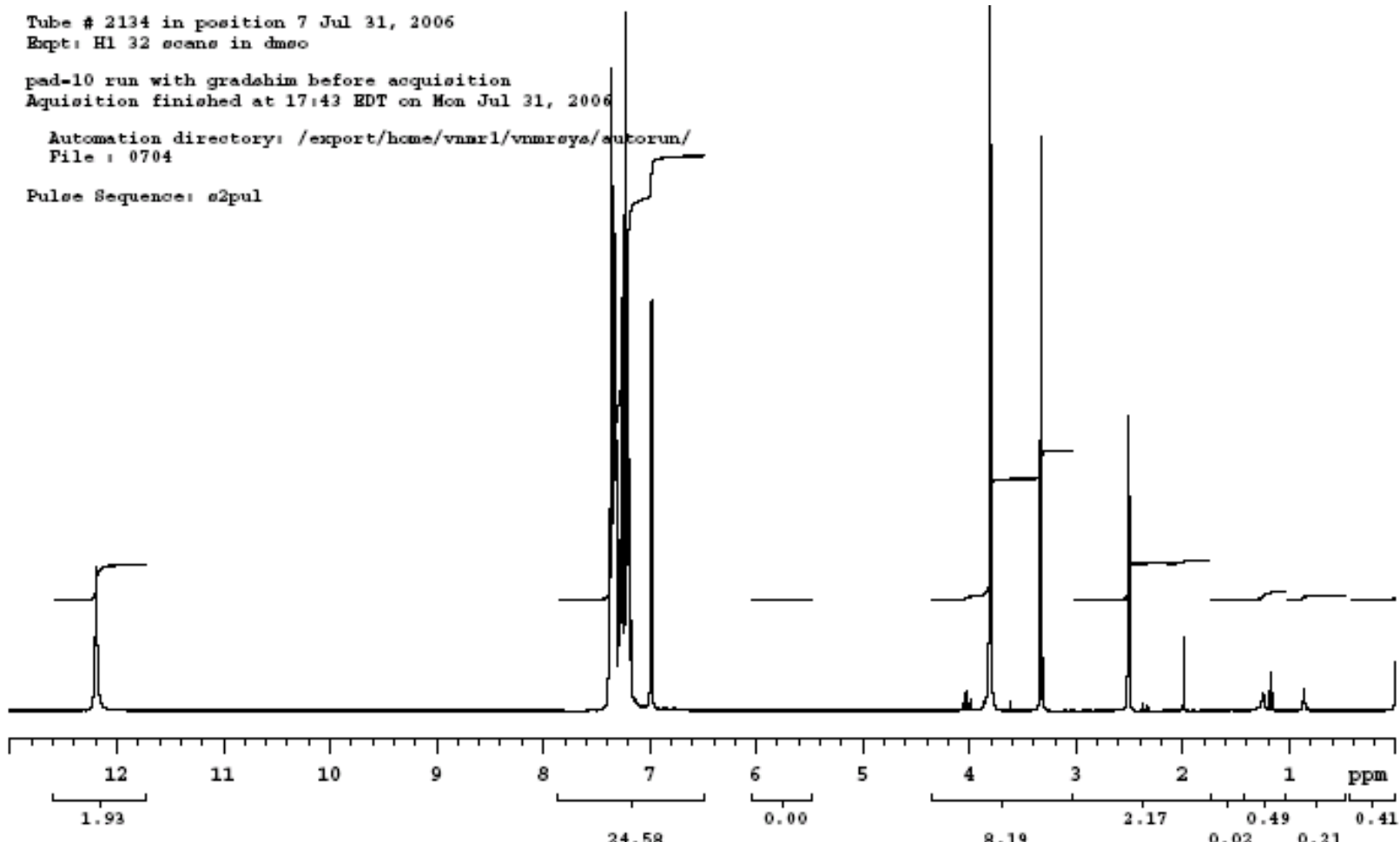

Tube \# 2134 in position 7 Jul 31, 2006 Bxpt, Quisk $\mathrm{C}_{13}$ in dmeo

Aquiaition finiohed at 17,48 BDT on Hon Jul 31, 2006

Automation directoryi/export/hcmo/vanr1/vnmreys/autorun/ File 10705

Puleo Sequenoel a2pul

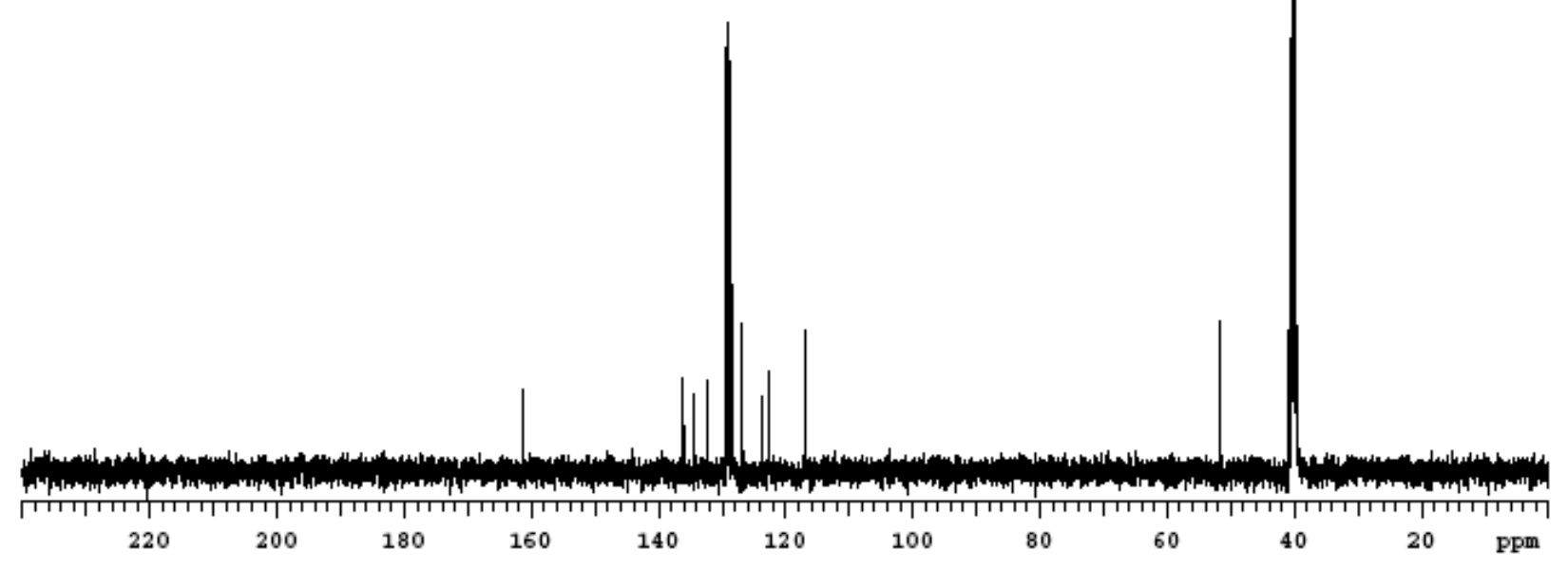


<smiles>[CH]C(C)=O</smiles>

Tube \#2354 in position 4 Aug 07, 2006

Bopt, H1 32 ecane in dmeo

pad-10 run with gradahim before acquiaition

Aquiøition finiøhed at 15,54 BDT on Hon Aug 07, 2006

Puloo Sequencol o2pul

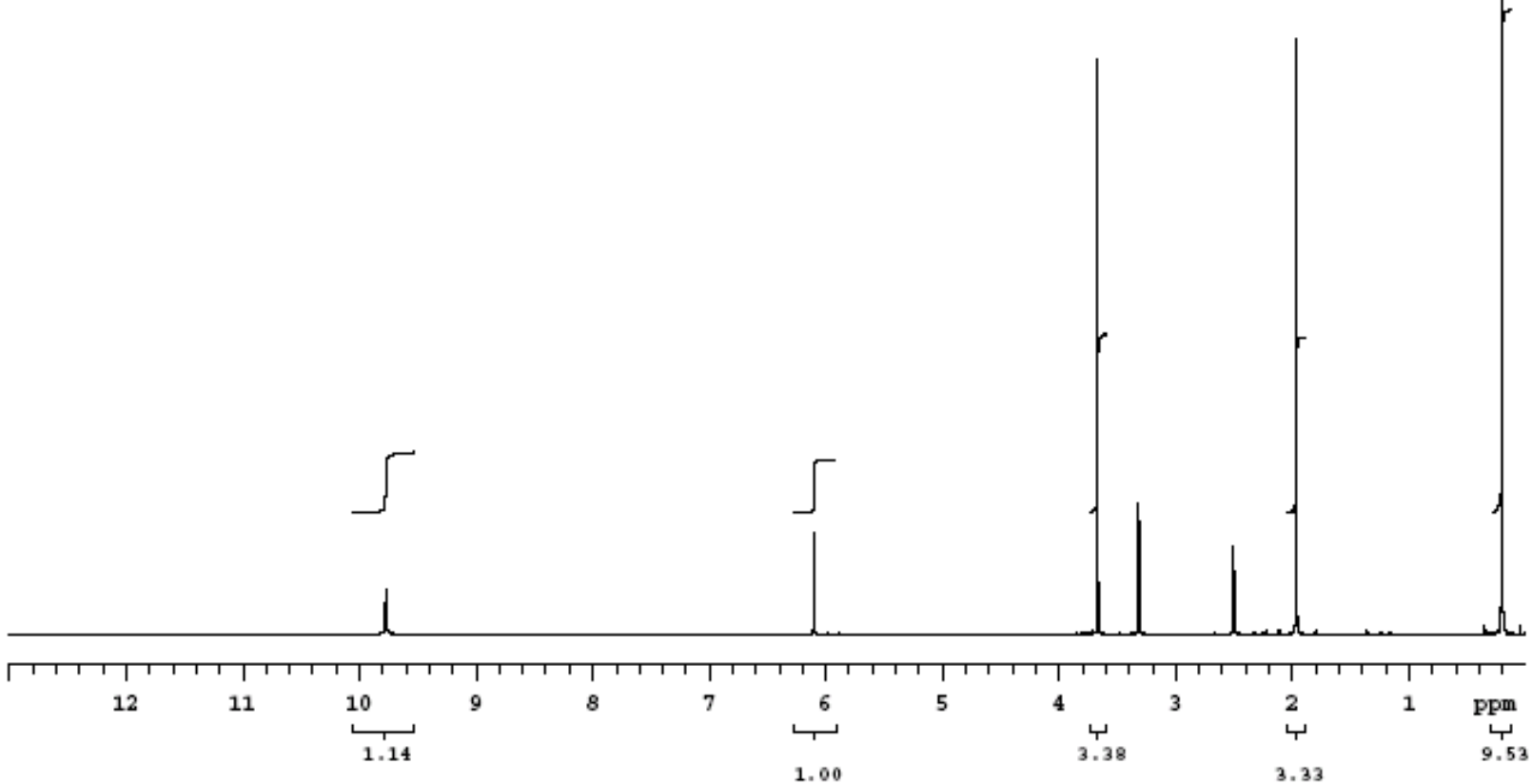

Tube \$2354 in position 4 Aug 07, 2006

Bopt I Quiok $\mathrm{Cl}_{13}$ in dmeo

Aquiaition finiohed at 15/59 BDT on Hon Aug 07, 2006

Automation directory!/export/hcme/vnnr1/vnmreys/autorun/ File , 0406

Puloo Sequenoel a2pul

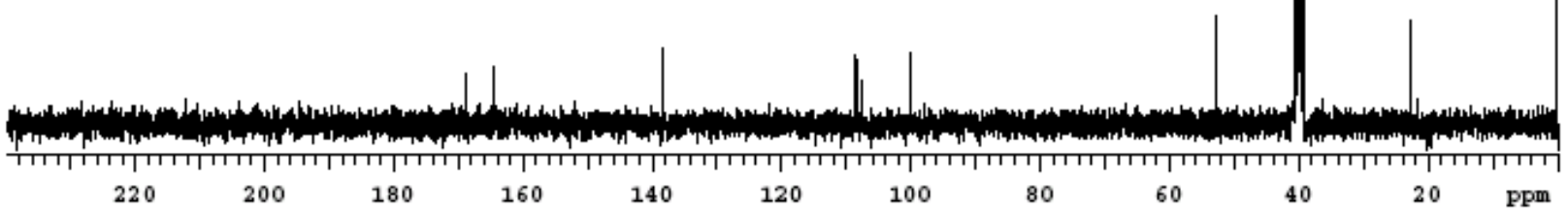


<smiles>COC(=O)c1cc(C(C)C)c(C)n1C(C)=O</smiles>

$\begin{array}{lll}1.5 & \mathbf{4 g} & 1.0\end{array}$

Tube \#2515 in position 19 Aug 11, 2006

Bopt I H1 32 voane in dmeo

pad-10 run with gradahim bofore acquiaition

Aquiaition finiahed at 11,33 EDT on Pri Aug 11, 2006

Automation directory'/export/hcme/vnmx1/vnmreys/autorun/

File I 1902

Puleo Sequenoel a2pul

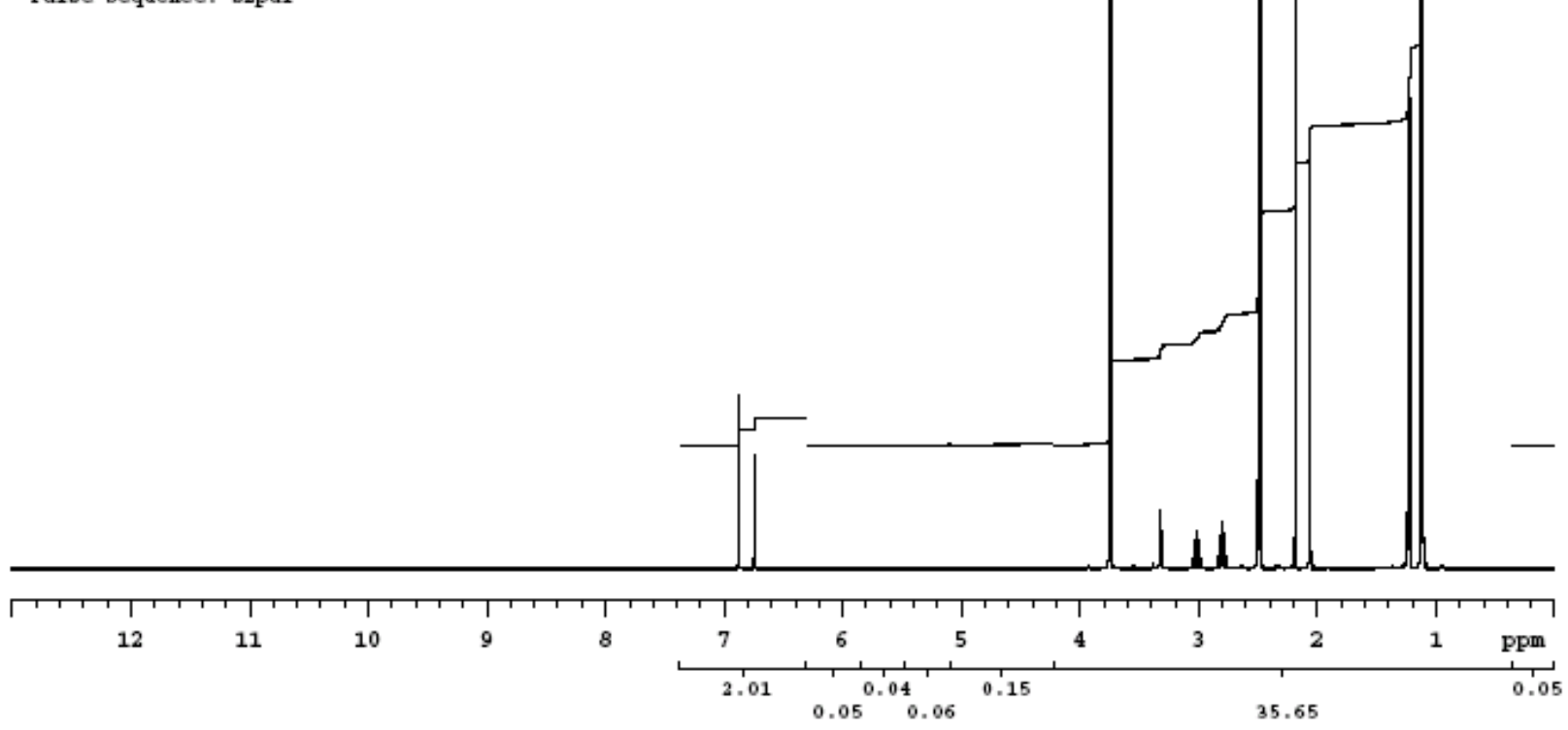

Tube \#2515 in position 19 Aug 11, 2006

Bopt I Quiok $\mathrm{Cl}_{13}$ in dmeo

Aquiaition finiohed at 11,37 BDT on Pri Aug 11, 2006

Automation directory1/export/homo/vnmr1/vnmreys/autorun/

File I 1903

Puloo Sequenoel o2pul

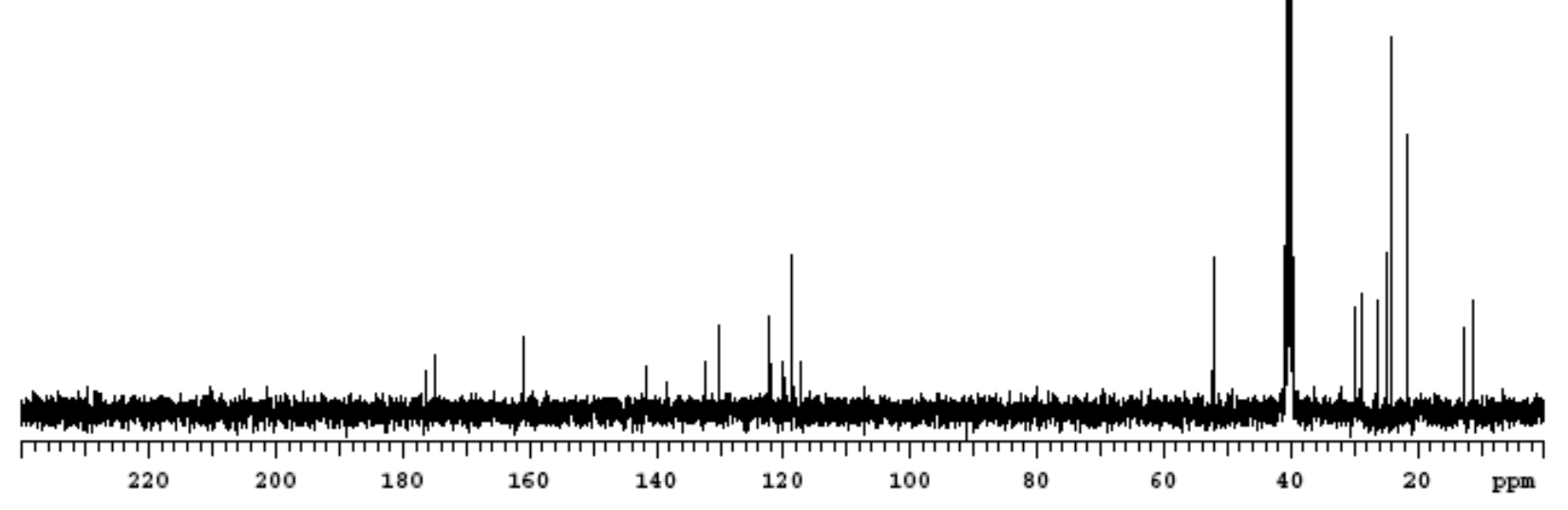


<smiles>COC(=O)c1cc(CO)c[nH]1</smiles>

$5 h$

Tube \#2322 in position 22 Aug 07,2006

Bspt I H1 32 ecane in dmeo

pad-10 run with gradahim bofore acquiaition

Aquiaition finiohed at 08,40 BDT on Hon Ang 07, 2006

Automation directory/ /export/home/vnmi/vnmxoys/autorun/

File , 2203

Pulao Sequencel o2pul

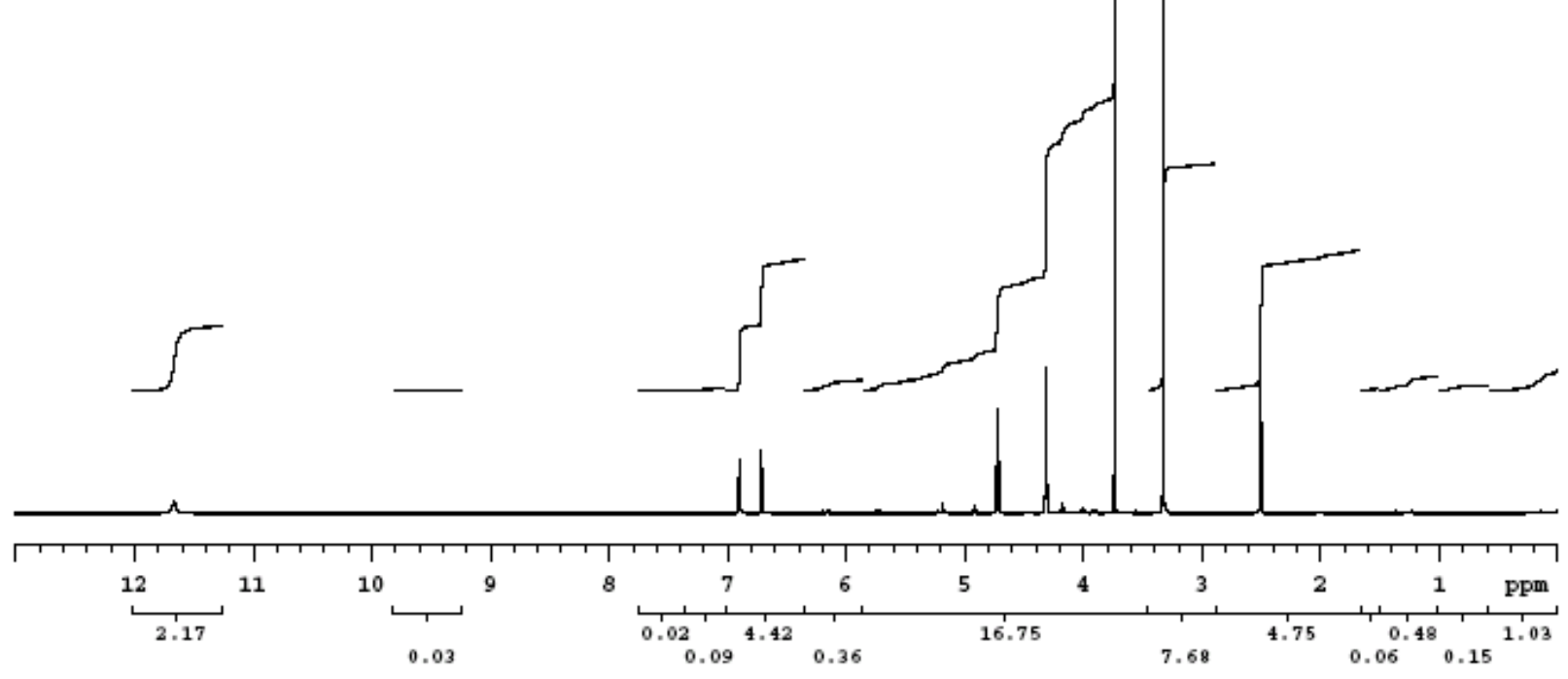

Tube \# 2322 in position 22 Aug 07,2006

Bopt, Quisk $\mathrm{C}_{13}$ in dmeo

Aquioition finiohed at 08,44 BDT on Kon Aug 07, 2006

Automation directoryı/export/homo/vnmx1/vnmroys/autorun/ File I 2204

Puloo Sequenoer a2pul

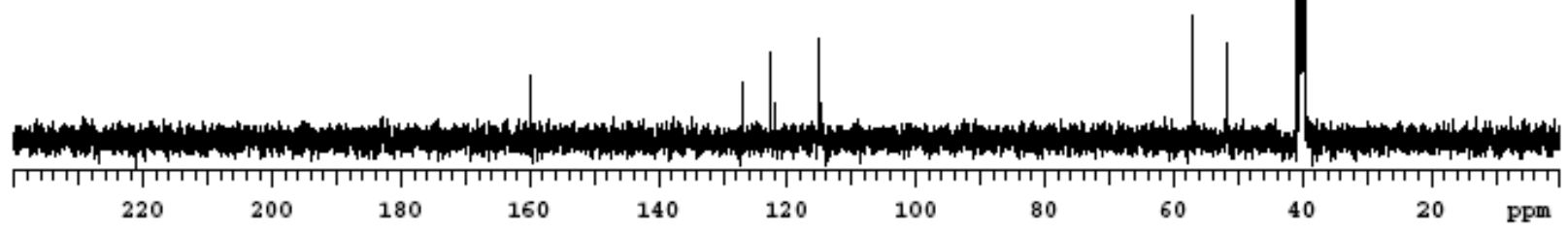




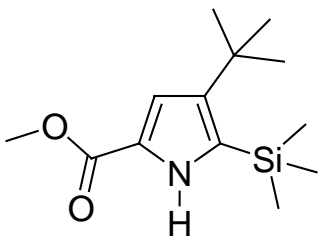

$5 \mathbf{i}$

00-407Z-INH ANA100-3040 CK\#34435

41072 o4a4 H1 OpenAcoese Data

Run Datel Auguot 07, $200601,14,48$

oldtest

arawlem A1972

Tube \# 2314 in position 14 Aug 05,2006

Bxpt, H1 32 scane in meo

pad-10 run with gradahim bofore acguiaition

Aquioition finiohod at 01,05 BDT on Kon Aug 07, 2006

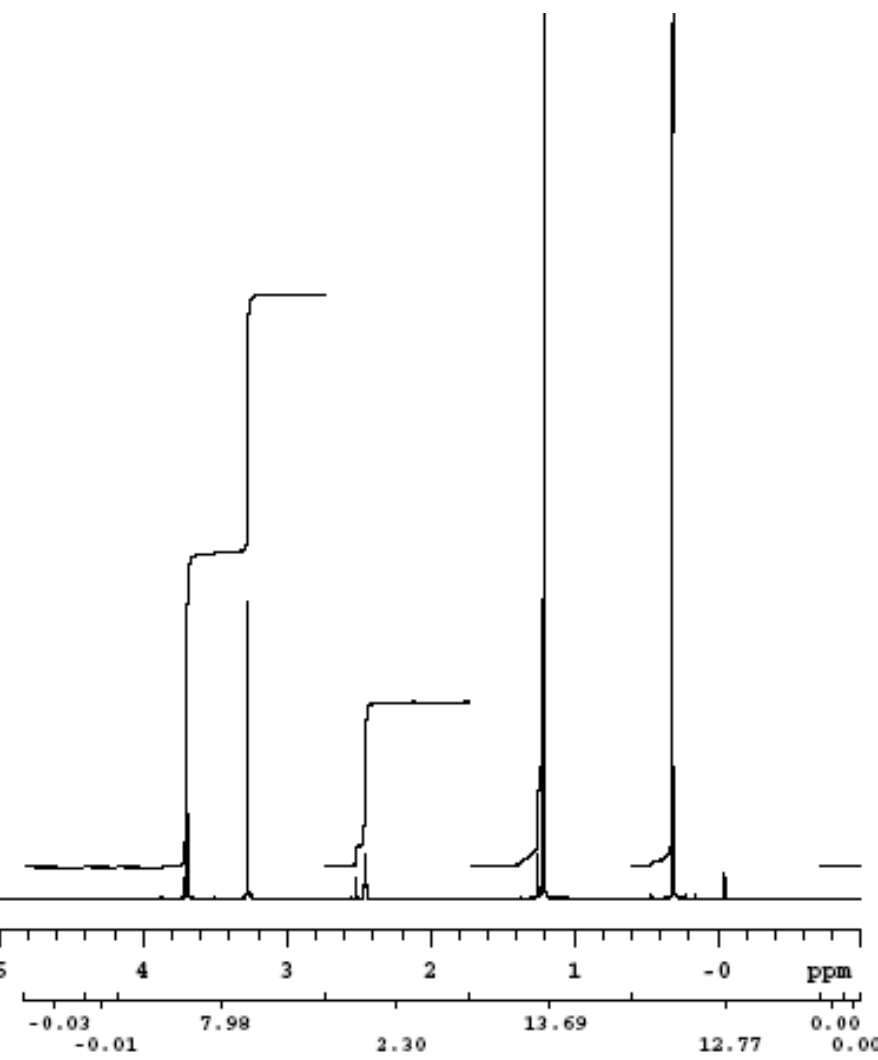

Tube \# 2314 in position 14 Ang 05,2006

Bapt I Quiok $\mathrm{C} 13$ in dmeo

Aquiaition finiohed at 01,09 EDT on Kon Aug 07, 2006

Automation direotoryı/export/home/vnme1/vnmroys/autorun/

File 1404

Puloo Soquenoel a2pul

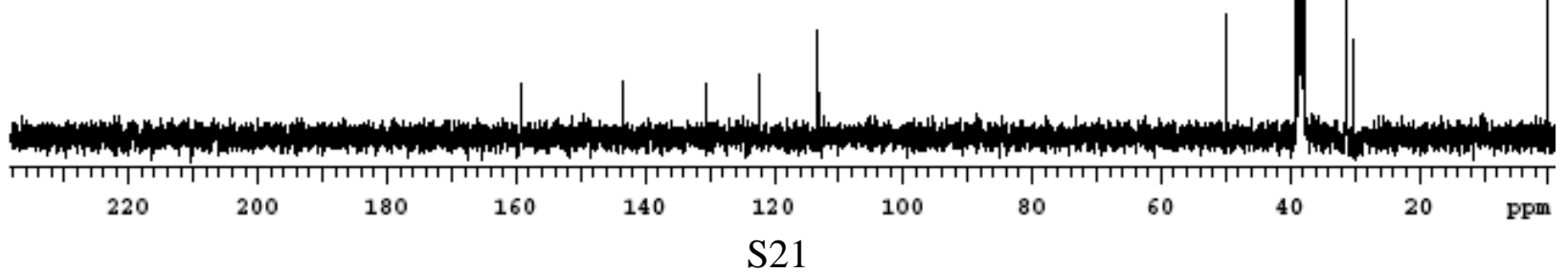




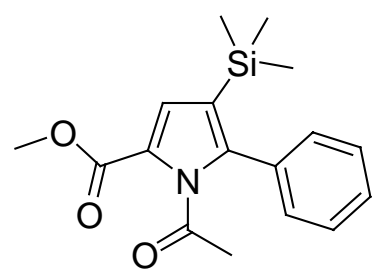

$4 \mathbf{j}$

Tube 2562 in position 16 Aug 14,2006 Bupt, H1 32 ecane in dmeo

pad-10 run with gradahim bofore acquiaition

Aquiaition finiahed at 17,09 BDT on Hon Aug 14, 2006

Puloe Sequenoel a2pul

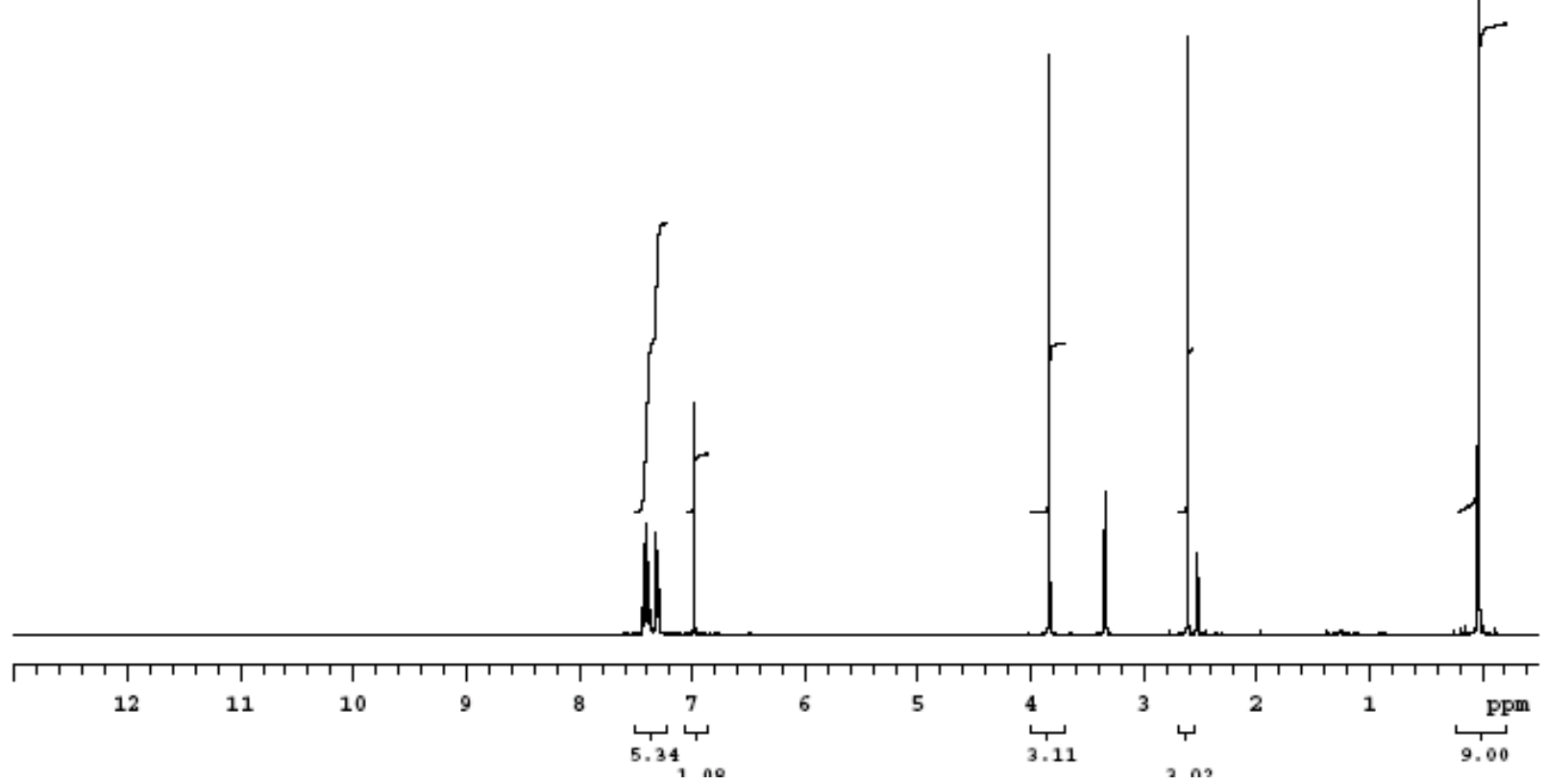

Tube \#2562 in position 16 Aug 14, 2006

Brpt I Quiok $\mathrm{C}_{13}$ in dmso

Aquiøition finiohed at 17,13 BDT on Kon Aug 14, 2006

Automation directory1/export/homo/vnmr1/vnmreys/autorun/

File I 1605

Puloo Sequenoel a2pul

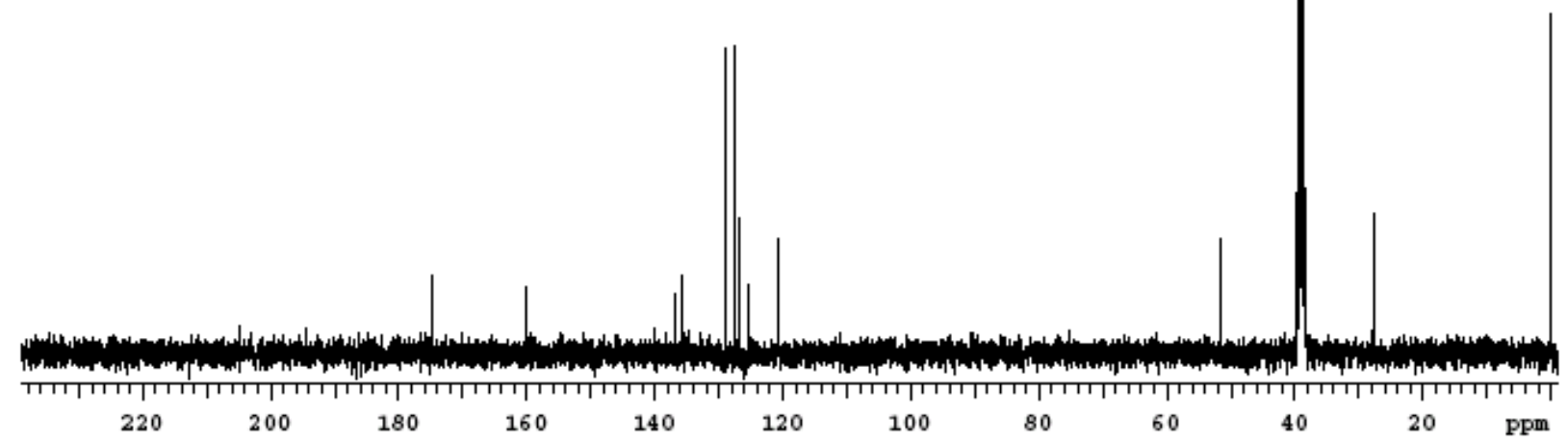




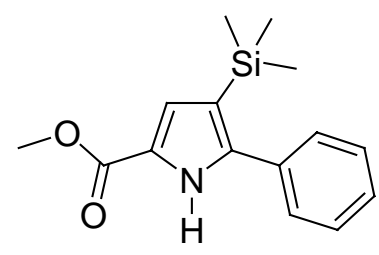

$5 j$

Tube \#2559 in position 12 Ang 14, 2006 Bopt, H1 32 ocane in dmeo

pad-10 run with gradshim before acquiaition

Aquioition finiohed at 16,26 BDT on Kon Ang 14, 2006

Pulve Sequenoel a2pul
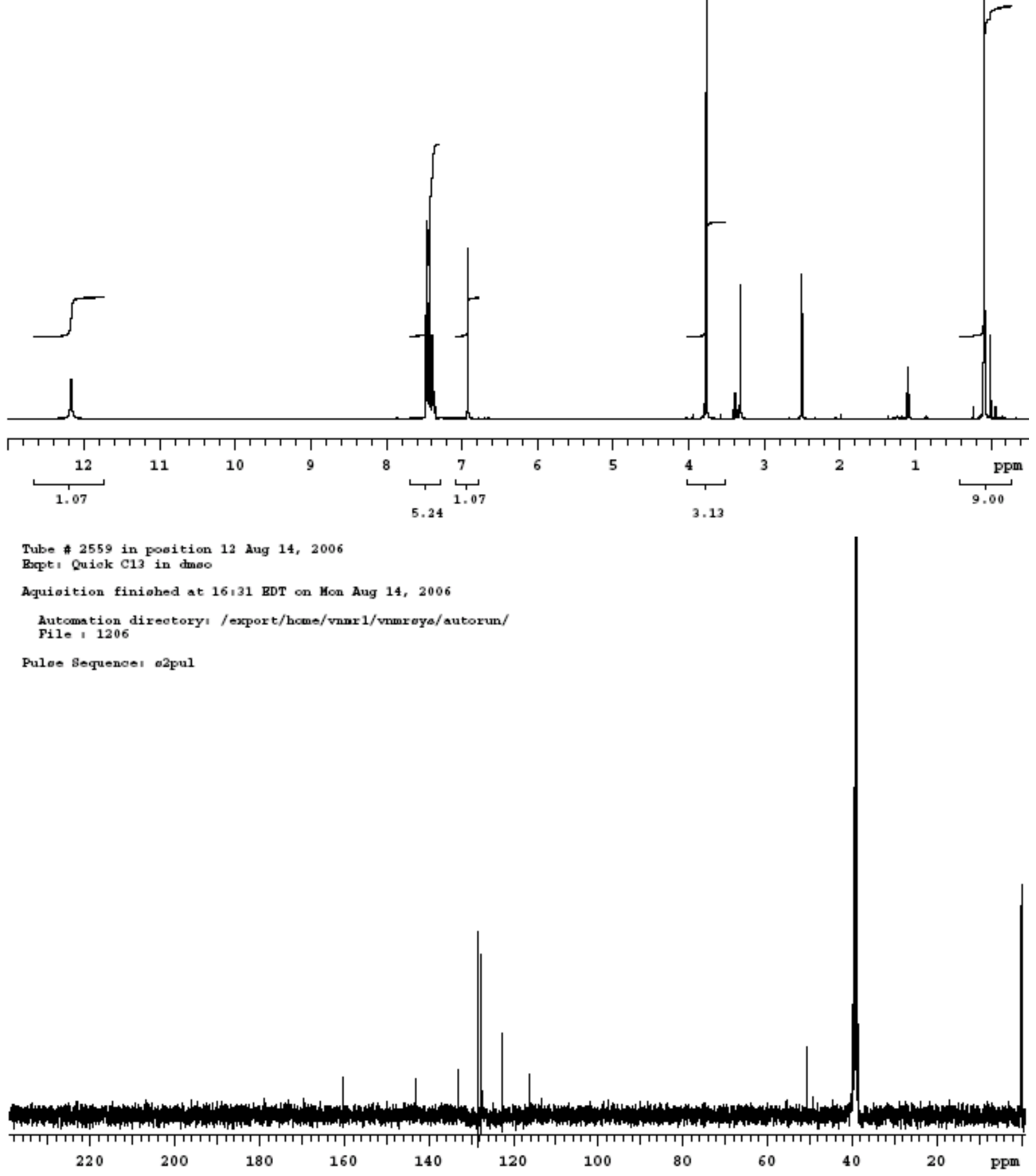


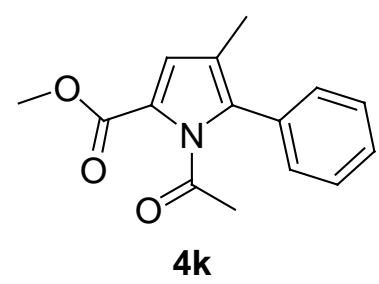

Tube \# 2046 in position $19 \mathrm{Jul} \mathrm{27,2006}$ Bapt, H1 32 ecane in dmeo

pad-10 run with gradahim bofore acquiaition

Aquioition finiohed at 15,55 BDT on Thu Jul 27, 2006

Automation directory।/export/hcme/vnmr1/vnmreys/autorun/ File, 1903

Puleo Sequenool a2pul

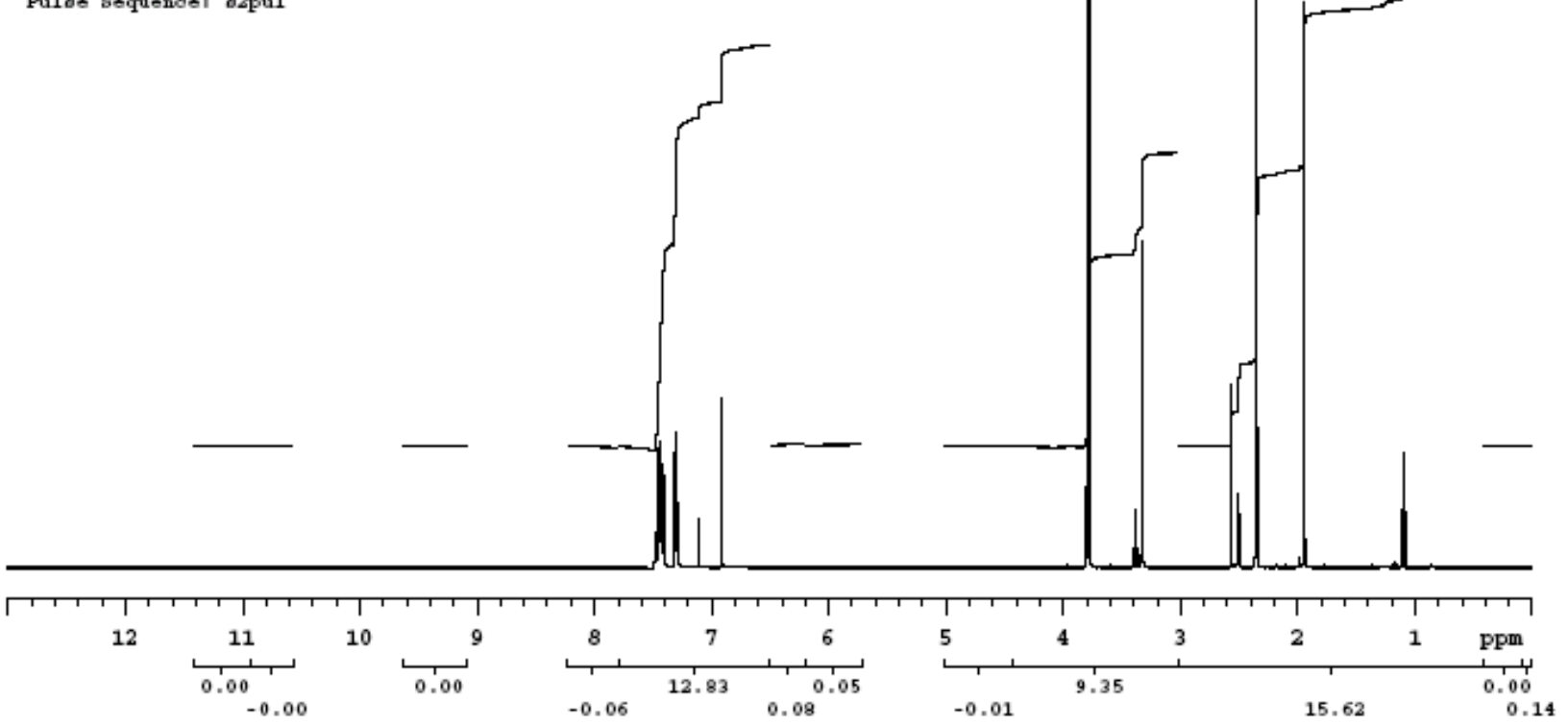

Tube \#2046 in position $19 \mathrm{Jul} \mathrm{27,2006}$ Brpt, Quisk $\mathrm{C}_{13}$ in dmeo

Aquiaition finiohed at 16,00 BDT on Thu Jul 27, 2006

Automation directory! /export/hcme/vnmr1/vnmreys/autorun/ File 1904

Puleo Sequenosi a2pul

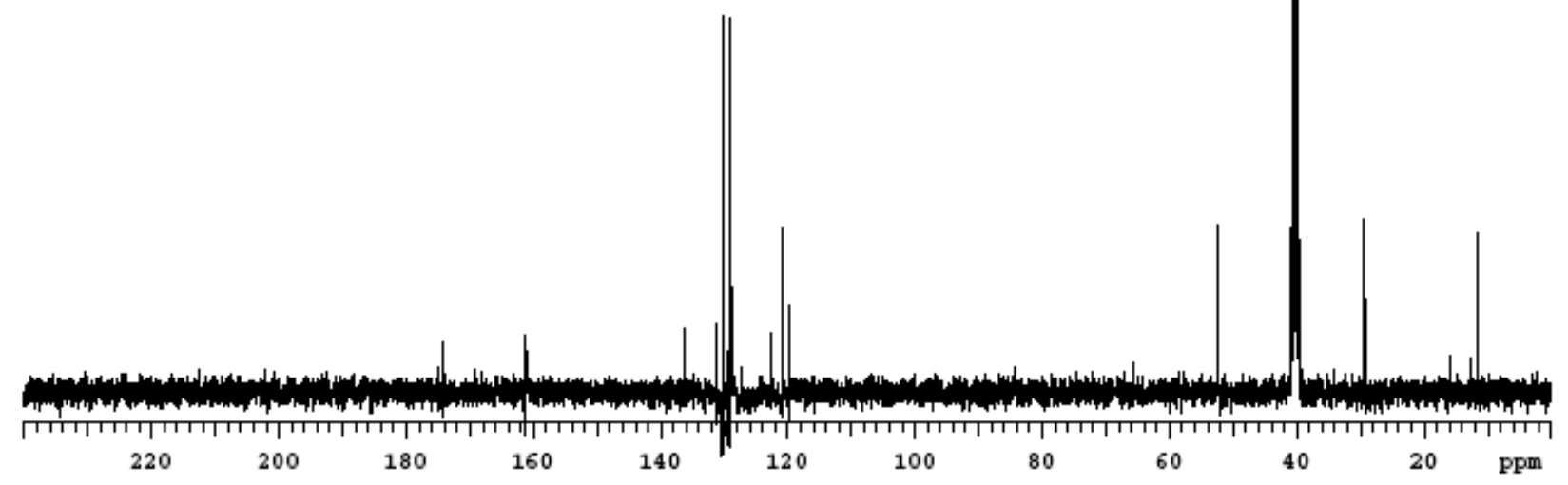




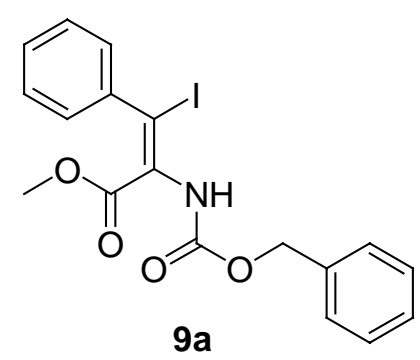

Tube \# 3245 in position 12 Sep 06,2006 Bopt, H1 32 ocane in odol3

pad-10 run with gradahim bofore acquiaition Aquiaition finiohed at 13,18 BDT on Wod Sep 06, 200 Automation directory1/export/homo/vnnr1/vnmreys/storun/ File, 1203

Puloo Sequenool a2pul

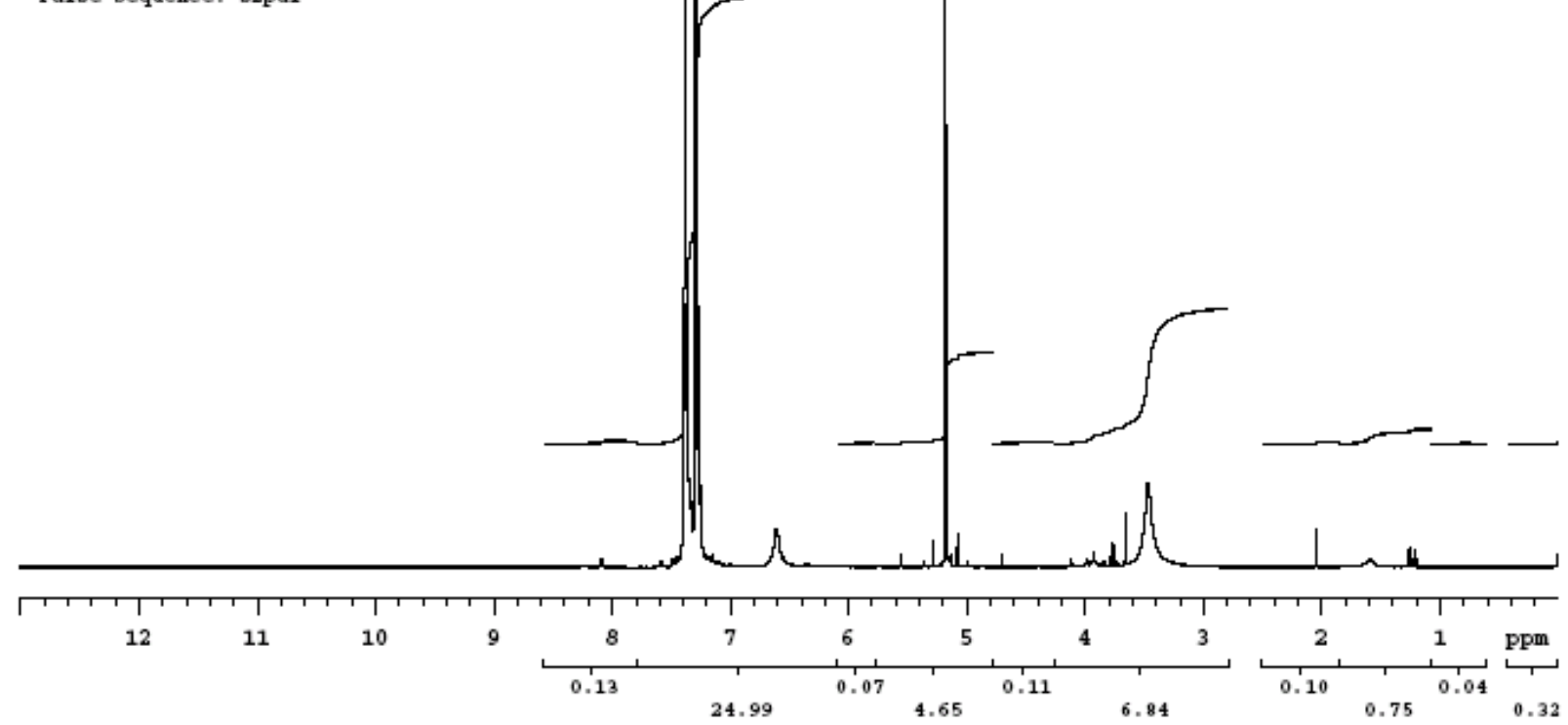

06-4771-INC AN\#I06-4174 CR\#351761 7877 o4a3 C13 on Varian IHOVA 400

Arohive Datel Sep 12 06:08131

Automation directoryı/export/homo/vmm 1/vm/royo/autorum/ File, 6303

Puloo Sequencor a2pul
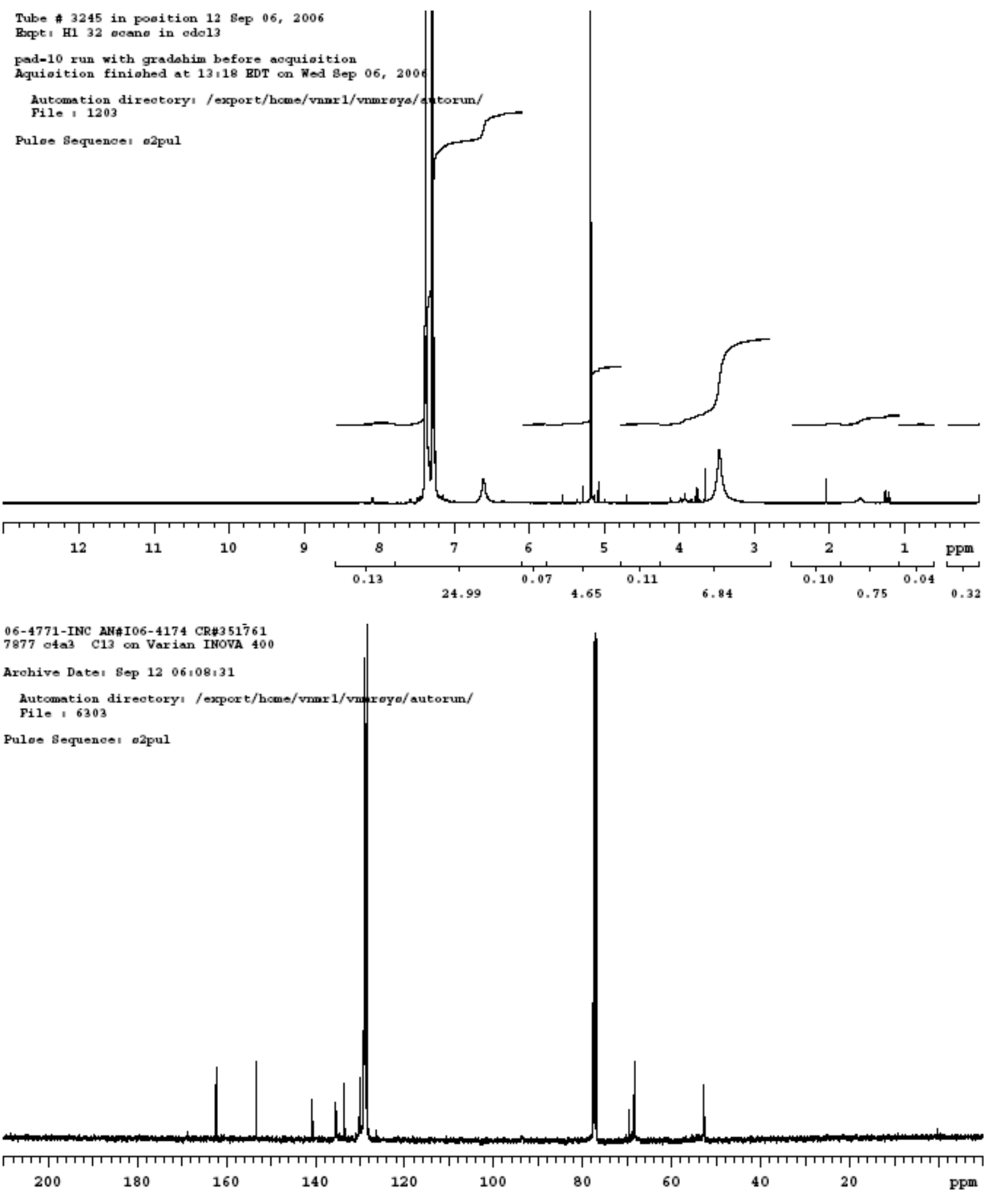


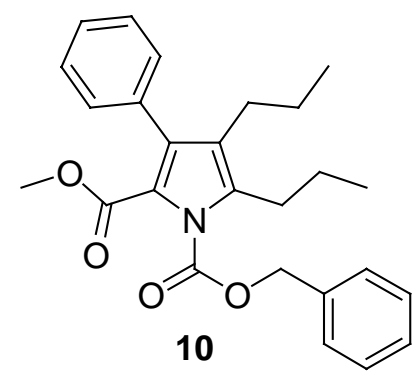

Tube \# 3274 in position 16 Sep 06,2006

Brpt , H1 32 ecane in dmeo

pad-10 run with gradahim before acquiaition

Aquiaition finiohed at 18,28 BDT on Wod Sep 06, 2006

Automation directory1/export/hcmo/vnnx1/vnmreys/autorun/

File I 1604

Puleo Sequenoel a2pul

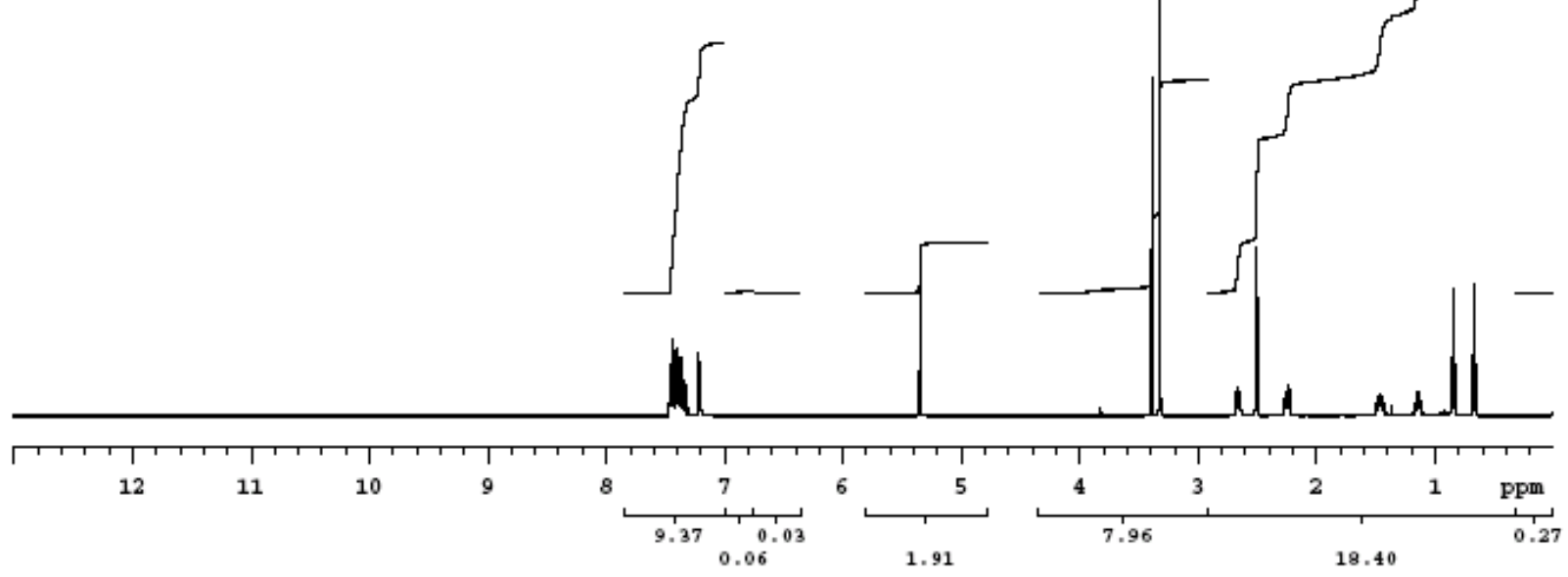

06-4713-INC AN\#I06-4190 CR\#351834 7521 o $4 a^{3}$ C13 on Varian INOVA 400

Arohivo Datel Sep $809,13,57$

Rephaved, $1 \mathrm{~b}=0.25 \mathrm{~Hz}$ to oee two

methyla on the propyl ohaine

Archivo Datol Sop $8,10,58,43$

Puloo Sequencer o2pul

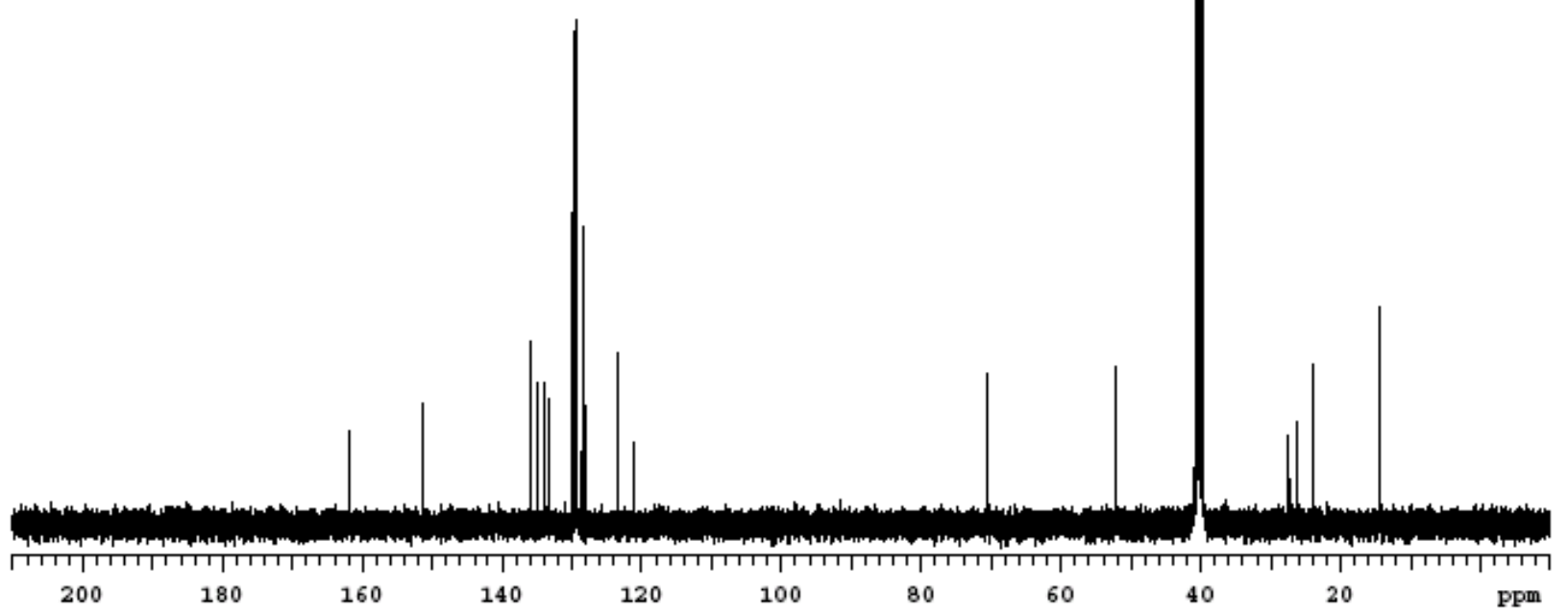




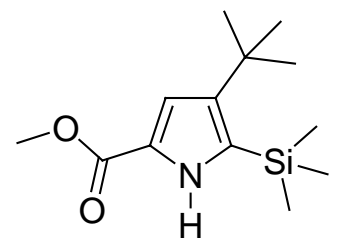

$5 i$

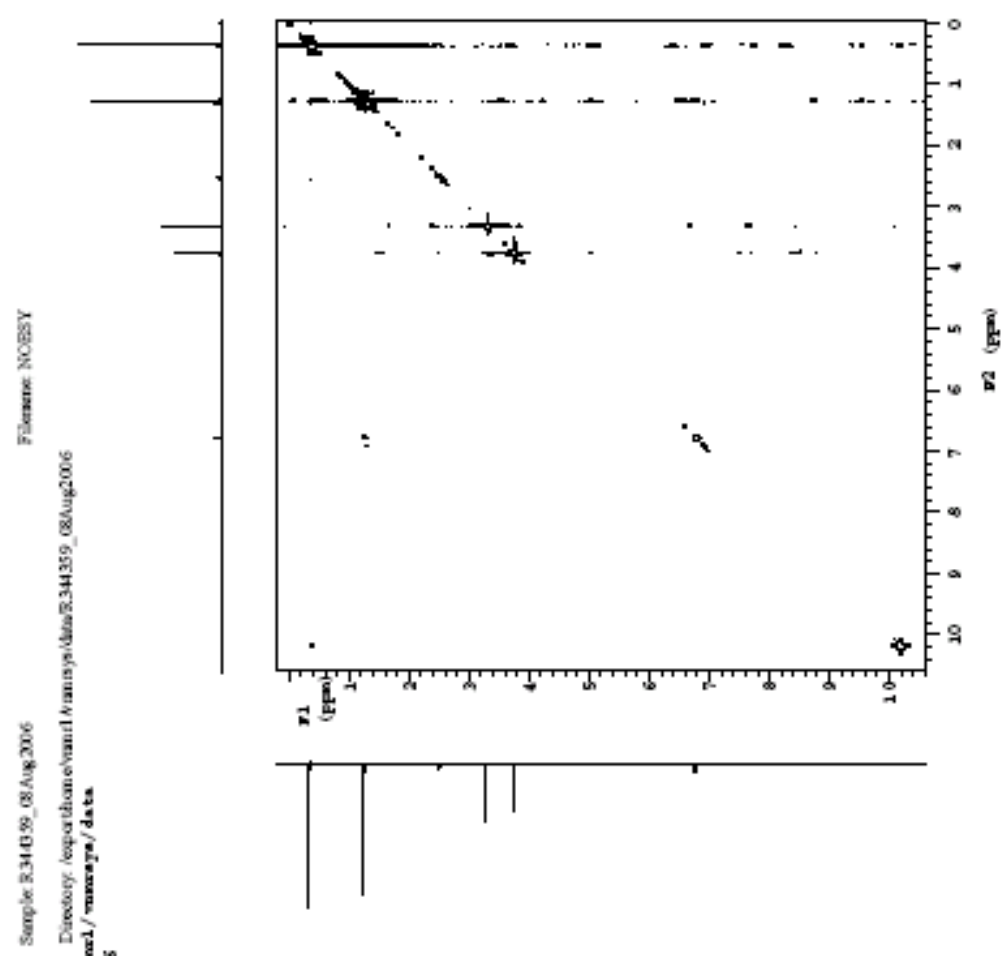




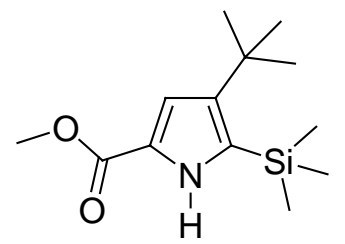

$5 i$

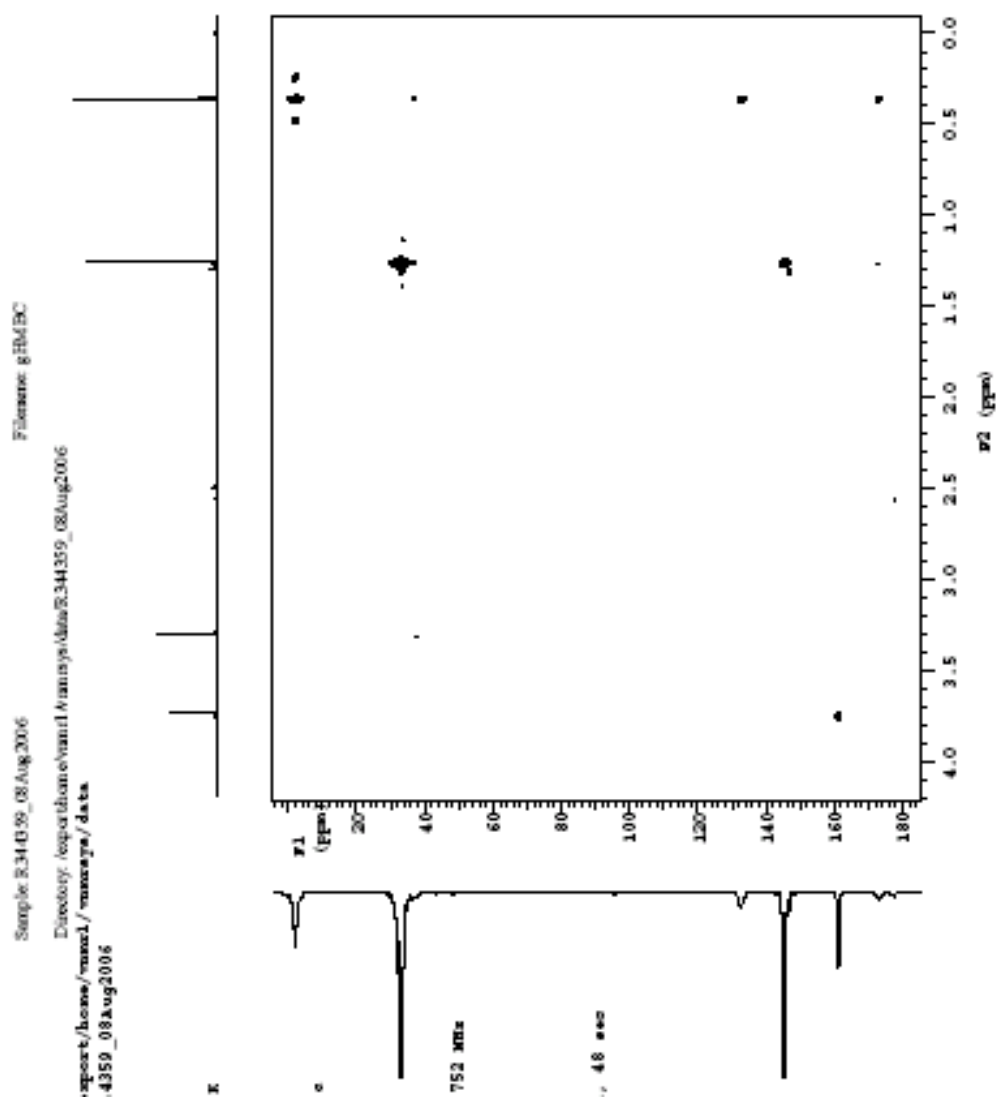




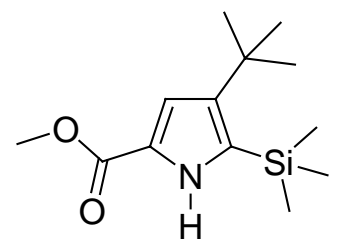

$5 \mathbf{i}$

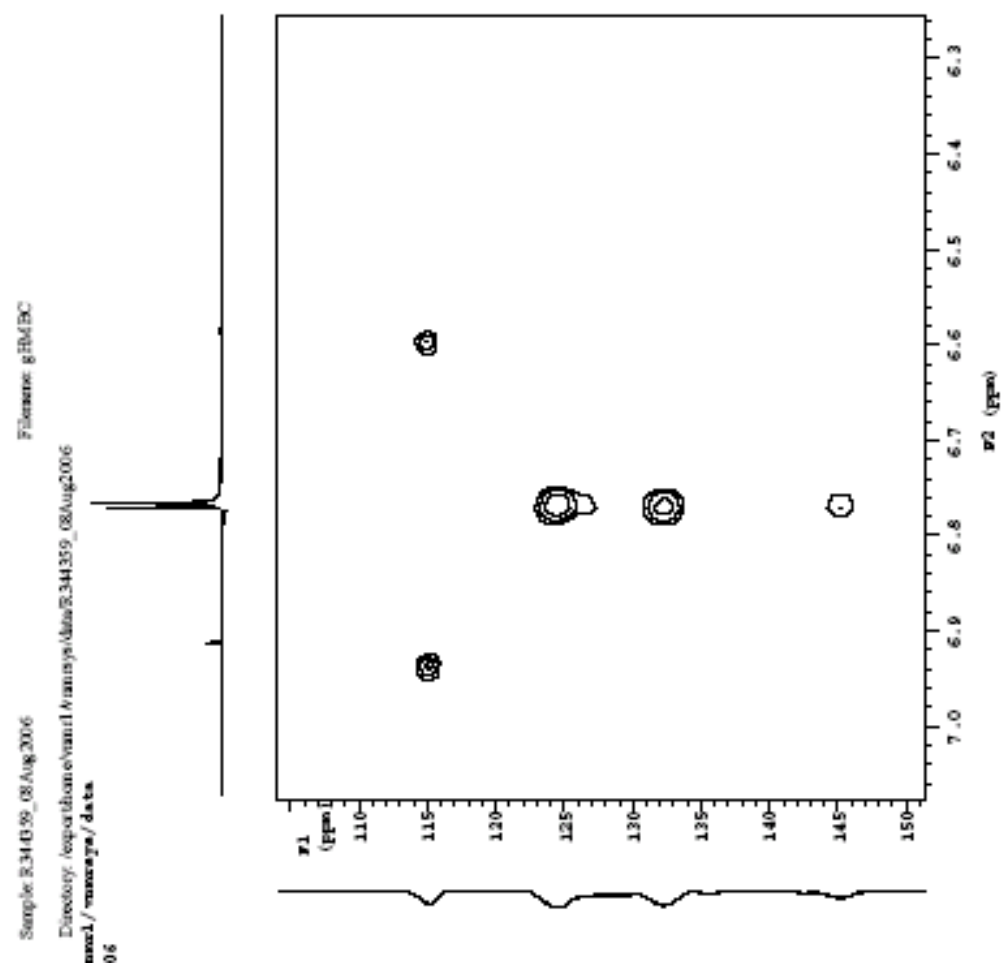




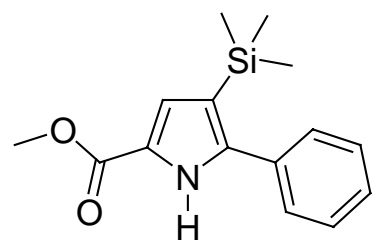

5j

Analytical\# I06-3810

Logbook\# 06-4927-INZ

Analyst IG

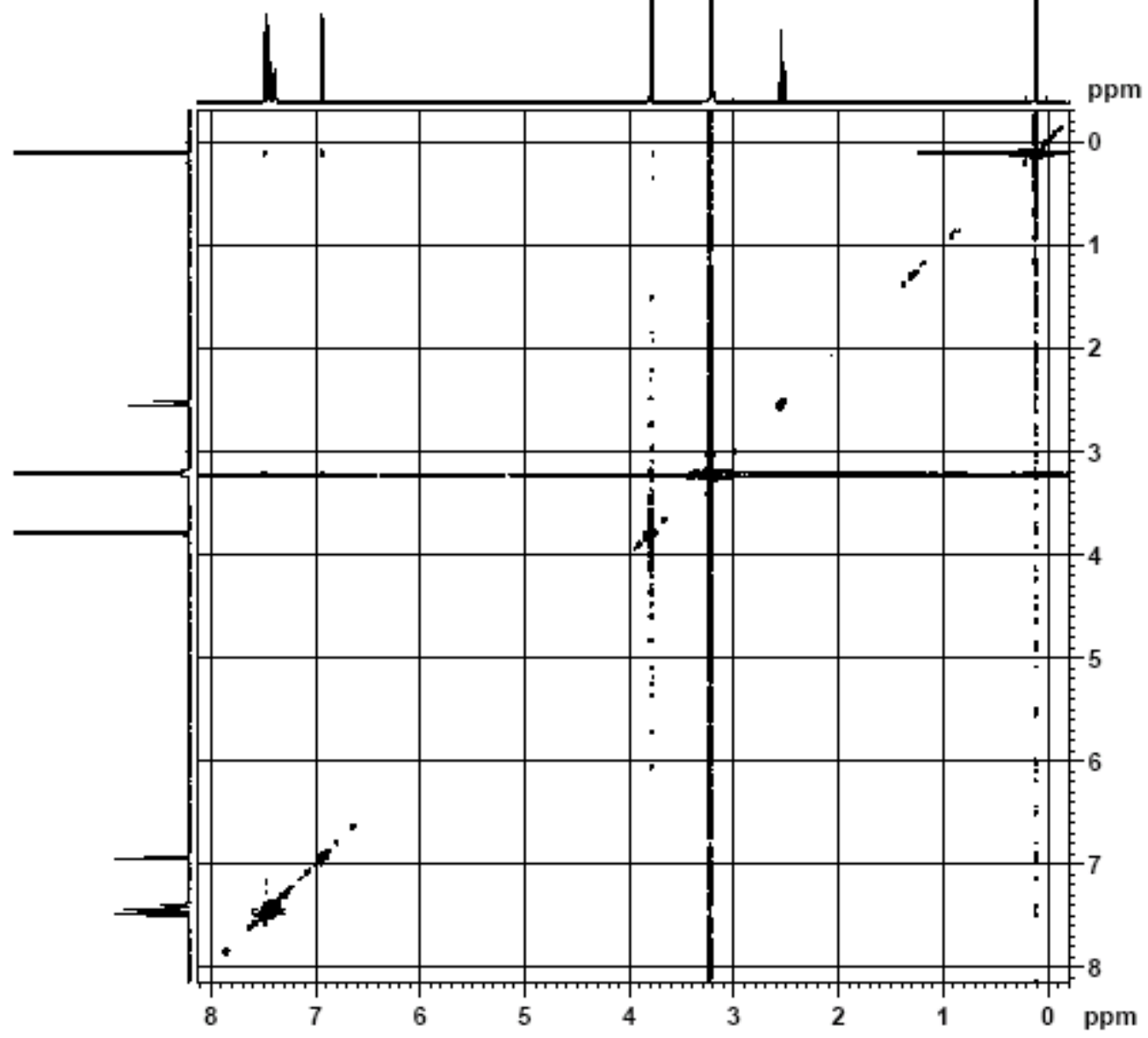




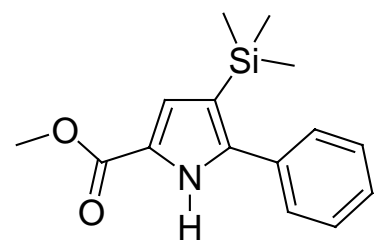

$5 j$

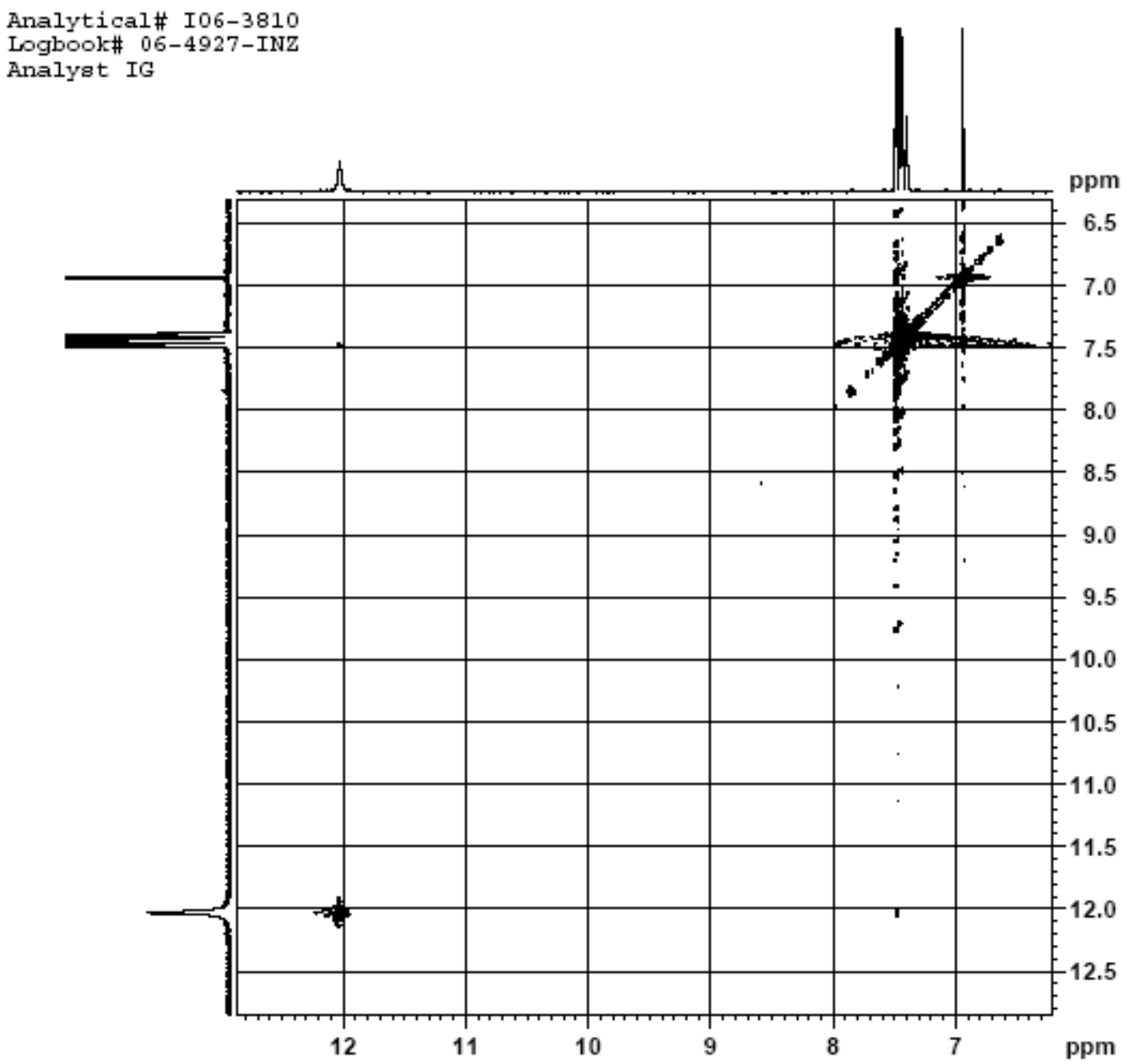


<smiles>COC(=O)c1cc([Si](C)(C)C)c(-c2ccccc2)[nH]1</smiles>

5j

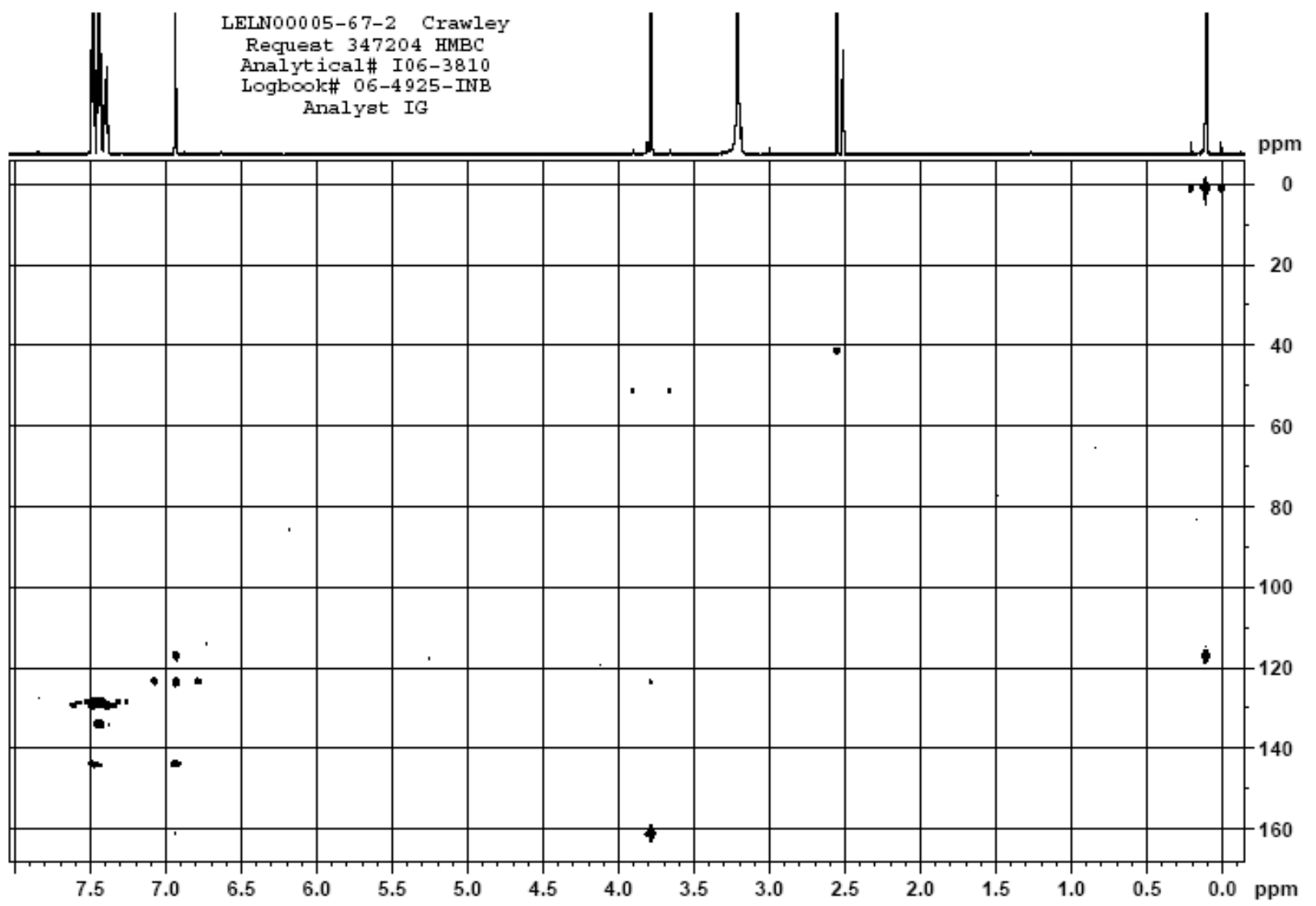




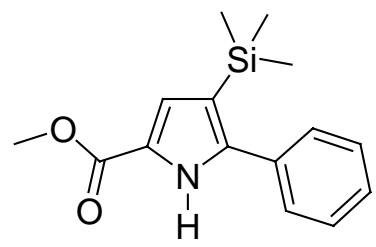

$5 j$

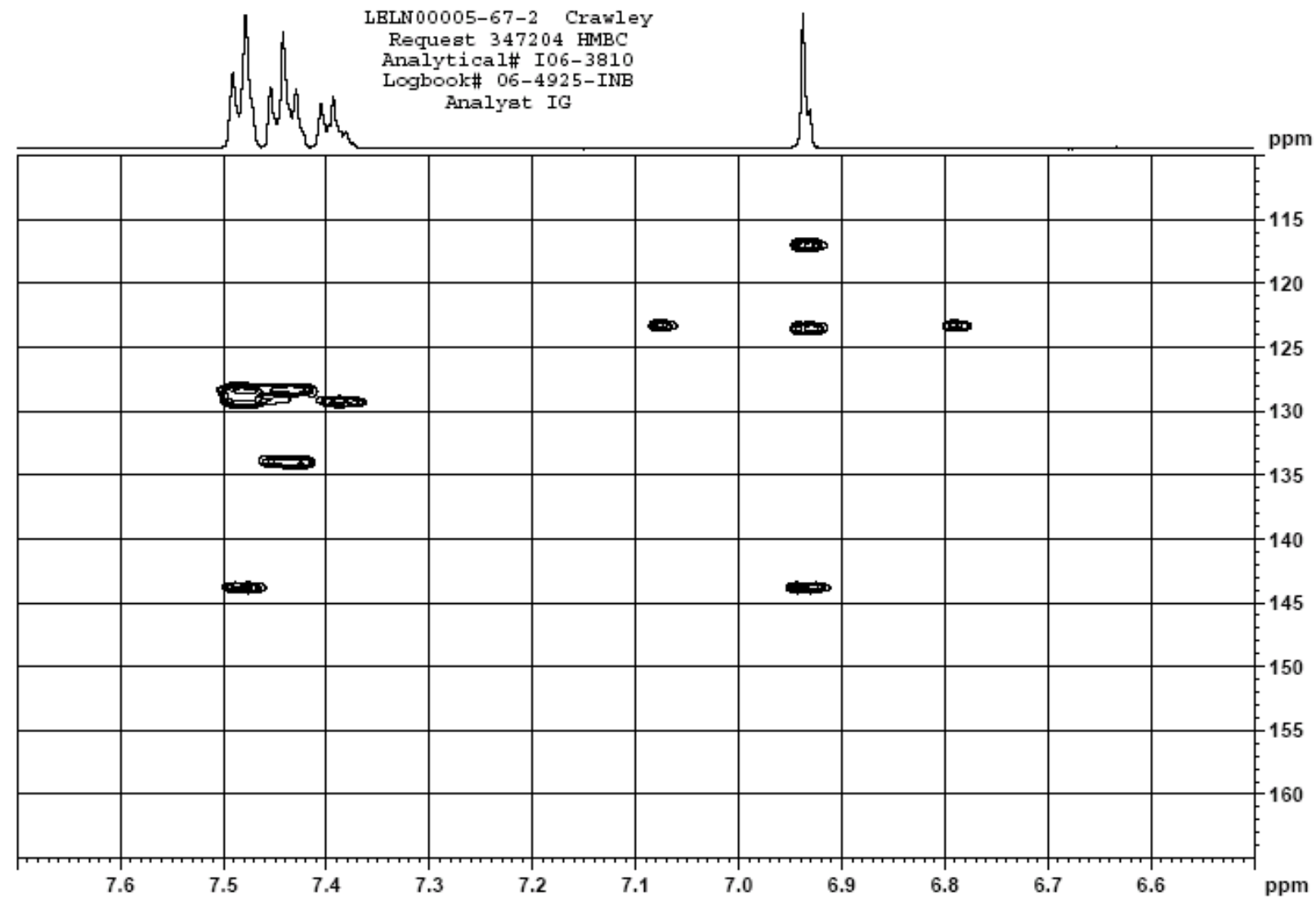




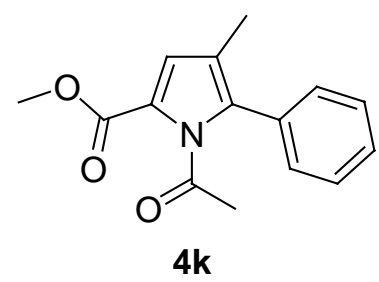

Sumple: R342504_08Aug2006

Filensme: NOESY

Directory: /export home/vanr1/vanrsys/data/R342504_08.Aug2006 nx1/vnnoyo/data
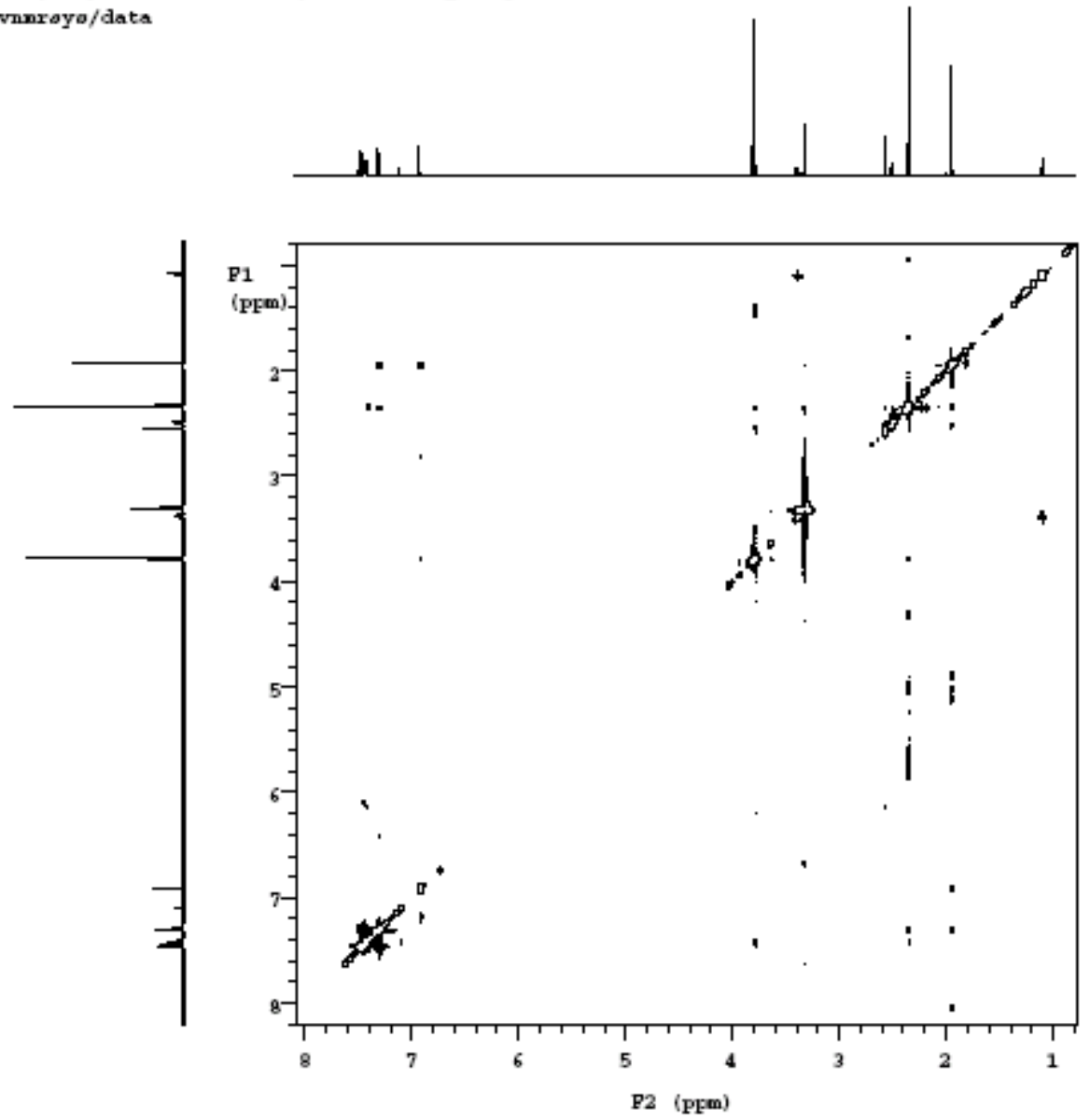


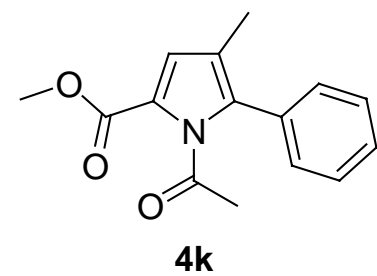

Sample: R342504_08.Aug2006

Filensme: gHMBC

Directory: /export home/vamrl/vanrsys/data/R342504_08.Aug2006 port/home/vmnx 1/vnnroyo/data 504_08Aug2006
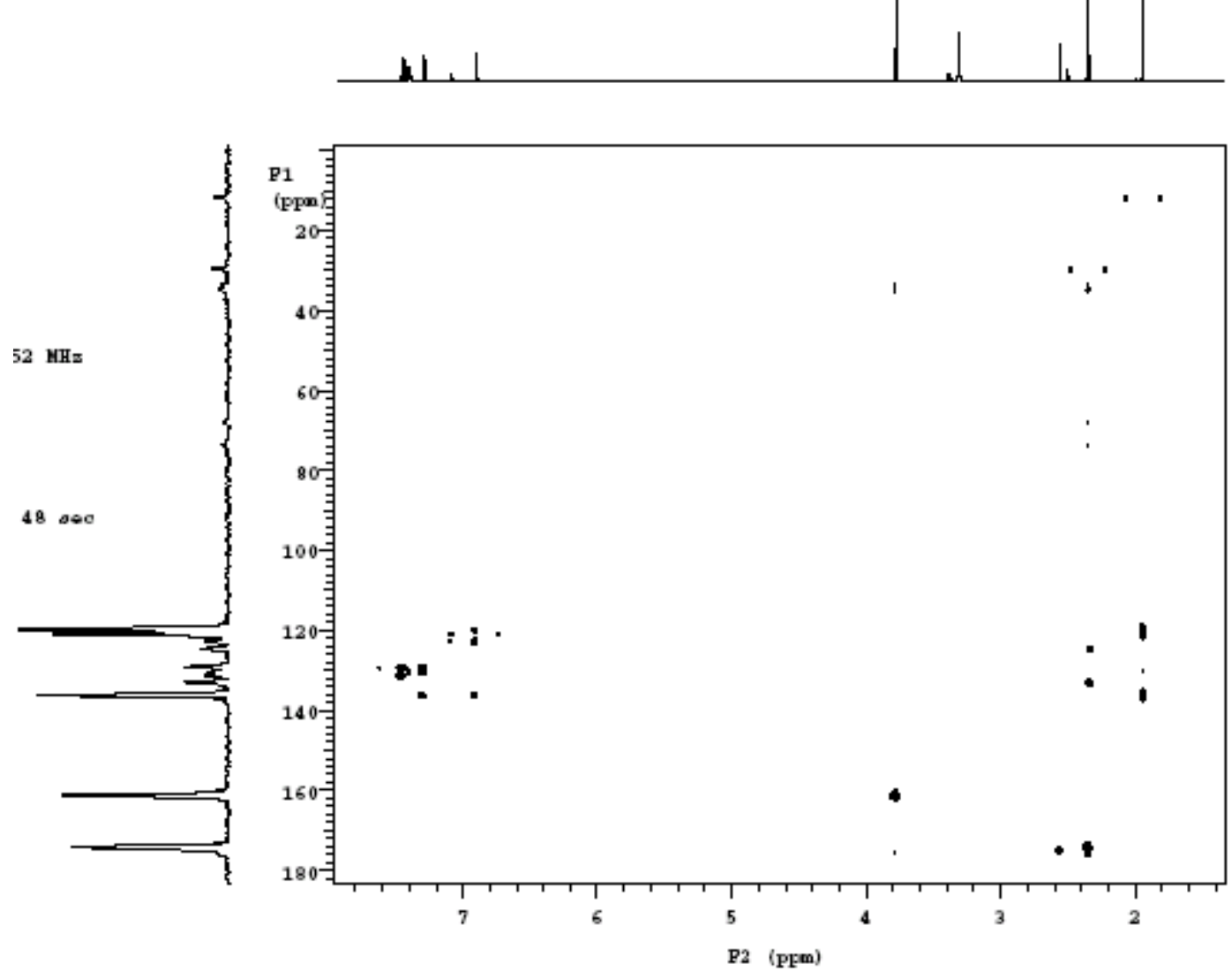


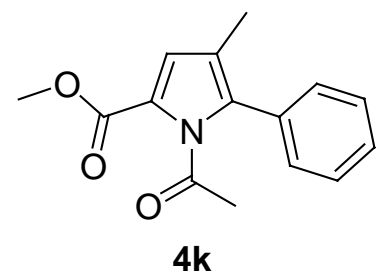

Sample: R342504_08Aug2006

Filensme: gHSQC

Directory: /export home/vunrl/vanrsys/data/R342504_08.Aug2006 xport/home/vmnx 1/vnnroyo/data

2504_08Aug2006

$\mathbf{R}$
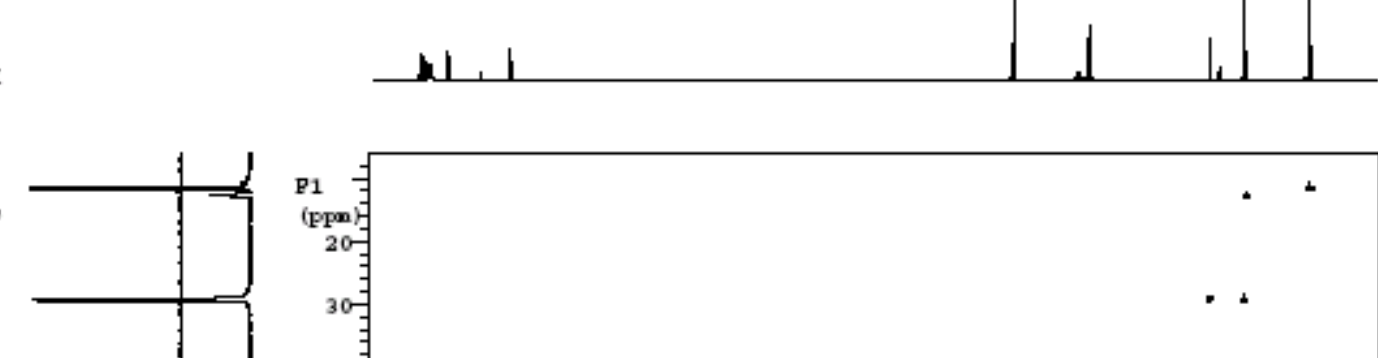

$752 \mathrm{NHz}$
$310 \mathrm{NHz}$

a

79 o00

19000

$\infty$

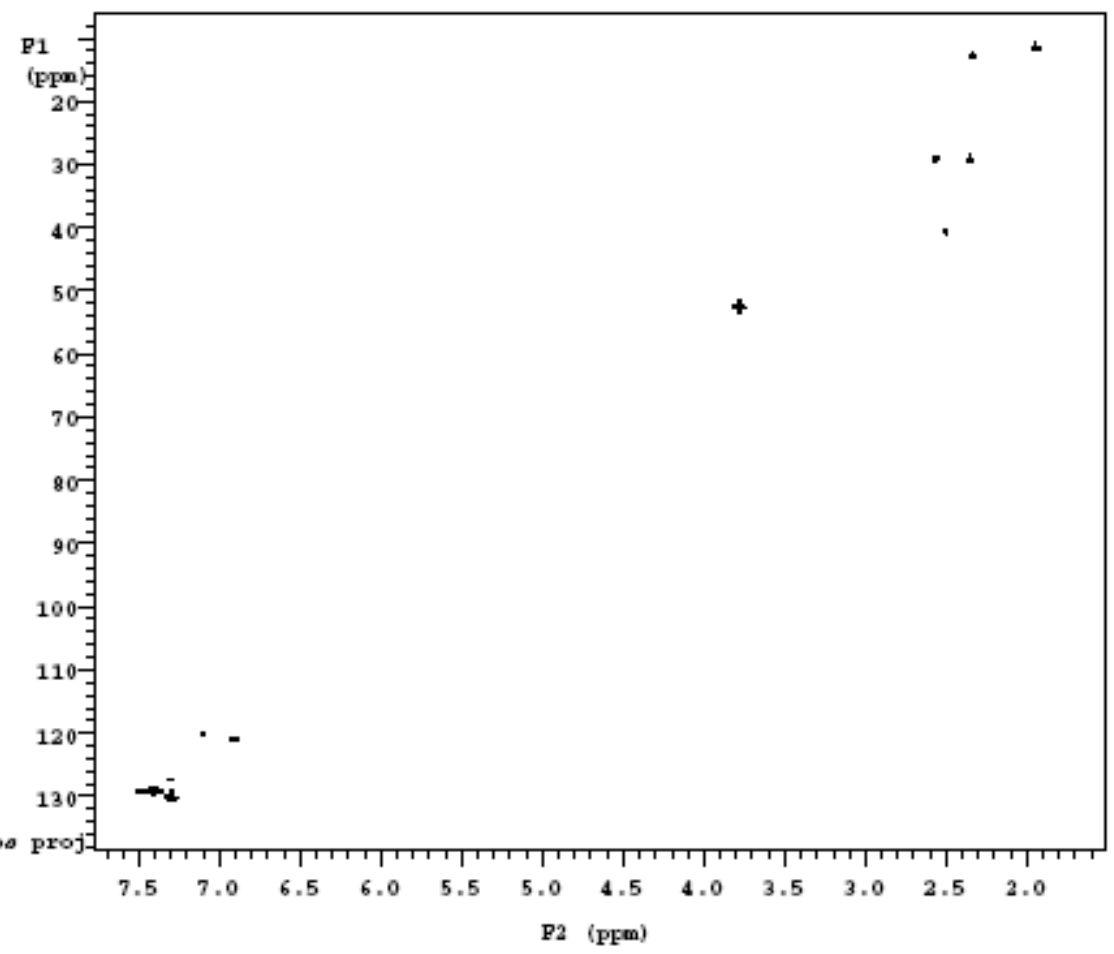

